\title{
ON THE NON-OCCURRENCE OF THE LAVRENTIEV PHENOMENON
}

\author{
GIOVANNI BONFANTI AND ARRIGO CELLINA
}

\begin{abstract}
We show that the Lavrentiev's phenomenon does not occur for functionals of the form

$$
\int_{\Omega} L(|\nabla u(x)|) d x
$$

where $L$ is an arbitrary convex function, provided that both $\partial \Omega$ and $u^{0}$ are of class $C^{2}$.
\end{abstract}

\section{INTRODUCTION}

In 1927 a remarkable paper by N. Lavrentiev [7] presented an example of a variational functional over the interval $(a, b)$, with boundary conditions $u(a)=\alpha$, $u(b)=\beta$, whose infimum over the set of absolutely continuous functions was strictly lower than the infimum of the same functional over the set of Lipschitzean functions satisfying the same boundary conditions. Since then, this phenomenon is called the Lavrentiev phenomenon. In 1934, B. Manià published a simpler example of this phenomenon [8] and, in 1993, Alberti and Serra Cassano [1] did show that the phenomenon does not occur for autonomous integrands over a one-dimensional integration set.

When the integration set is a subset $\Omega$ of $\mathbb{R}^{N}$, the boundary condition is described by the inclusion $u-u^{0} \in W_{0}^{1,1}(\Omega)$ and, in order for the problem of the occurrence of the Lavrentiev phenomenon to make sense, $u^{0}$ is a Lipschitzean function on $\bar{\Omega}$; in section 5 we present a modification of Manià's functional on $\Omega \subset \mathbb{R}^{2}$ with a linear boundary function $u^{0}$, exhibiting the Lavrentiev phenomenon. Connections between the regularity of a solution and the non-occurrence of Lavrentiev's phenomenon have been pointed out in [5]. An exhaustive literature on the Lavrentiev phenomenon can be found in [2].

In [3], Lemma 2.1, Esposito, Leonetti and Mingione prove that the phenomenon does not occur for functionals of the form

$$
\int_{\Omega} f(\nabla v(x)) d x
$$

provided that $\Omega$ is the unit ball, $f$ is a convex $C^{2}\left(\mathbb{R}^{N}\right)$ function and the growth of $f$ is of the $(p-q)$ type, i.e., $m|z|^{p} \leq f(z) \leq L(1+|z|)^{q}$, with $2 \leq q<p<2+q$; in addition, some further growth conditions on the first and second derivatives of $f$ are assumed.

The purpose of the present paper is to prove the following result, an approximation result that, in particular, guarantees the non-occurrence of the Lavrentiev phenomenon.

Theorem 1. Let $\Omega \subset \mathbb{R}^{N}$ be an open bounded set, with $\partial \Omega \in C^{2}$; let $u^{0} \in C^{2}(\bar{\Omega})$; let $L:[0, \infty) \rightarrow[0, \infty)$ be convex and such that $L(0)=0$. Let $u \in u^{0}+W^{1,1}(\Omega)$ be 
bounded on $\Omega$ and such that

$$
\int_{\Omega} L(|\nabla u(x)|) d x<\infty .
$$

Then, given $\varepsilon>0$, there exists $u_{\varepsilon} \in u^{0}+W^{1,1}(\Omega)$, with $u_{\varepsilon}$ Lipschitzean on $\bar{\Omega}$, such that

$$
\int_{\Omega} L\left(\left|\nabla u_{\varepsilon}(x)\right|\right) d x \leq \int_{\Omega} L(|\nabla u(x)|) d x+\varepsilon .
$$

The previous Theorem contains neither regularity nor growth assumptions on the Lagrangian $L$, besides its being convex.

In Manià's example, one reaches the conclusion of the existence of the Lavrentiev phenomenon by a rather long and clever computation. A much simpler computation, consisting in approximating the solution $x(t)=t^{\frac{1}{3}}$ by the Lipschitzean function

$$
x_{h}(t)=\left\{\begin{array}{l}
h t \text { for } 0 \leq t \leq h^{-\frac{3}{2}} \\
t^{\frac{1}{3}} \text { for } h^{-\frac{3}{2}} \leq t \leq 1
\end{array}\right.
$$

shows that, as the parameter $h \rightarrow+\infty$, the difference between the value of the integral functional computed on $x_{h}$ and the same integral computed on the solution, diverges to $+\infty$. This fact, although surprising, is not, by itself, sufficient to establish the validity of the Lavrentiev phenomenon. The proof of the non-occorrence of the Lavrentiev phenomenon that we present in this paper will be largely based on the following claim: if we are able to define a function $w_{h}$, analogous to the function $x_{h}$, issuing from the boundary datum in a "linear" way, such that, as the parameter $h$ diverges, the difference of the integrals computed along $w_{h}$ and along the solution, converges to zero, then the the Lavrentiev phenomenon does not occur. To show that the difference of the two integrals converges to zero, we will use the fact that an affine function is always a solution, among the function satisfying the same boundary conditions, of a convex variational problem depending only on the gradient. This fact is independent of any further regularity assumption on the Lagrangian $L$. Hence, we shall need regularity on the boundary datum $u^{0}$ to build the "linear" approximation, but we shall not need any regularity on $L$.

Finally, notice that, when $u$ is a solution, the boundedness of $u$ follows from the boundedness of $u^{0}$ under mild additional conditions [4].

\section{Notations AND PRELIMINARY RESUlTS}

We shall use the following notation. $B(x, \delta)$ is the open ball centered at $x$ of radius $\delta$. The Lebesgue measure of a subset $A$ of $\mathbb{R}^{N}$ is $|A| ; \omega_{N}$ is the measure of the unit ball; the complement of $\Omega$ is $C \Omega ; d(x)=\operatorname{dist}(x, C \Omega)$, a Lipschitzean function of Lipschitz constant 1; diam is the diameter of $\Omega ; \Omega_{\delta}=\{x \in \Omega: d(x) \leq \delta\}$. $d_{H}$ is the Hausdorff distance; the normal to $\partial \Omega$ at the point $y$, pointing towards the interior of $\Omega$, is $\nu(y) ; T(y)$ is the tangent plane to $\partial \Omega$ at $y$ and $T^{1}(y)=\{\tau \in T(y):|\tau|=1\}$. A vector $x \in \mathbb{R}^{N}$ will be often written as $\left(\hat{x}, x_{N}\right)$. The Hessian matrix of a function $\phi$ is $H_{\phi}$. For the coarea Theorem and the notion of Jacobian of a map $g: \mathbb{R}^{N} \rightarrow \mathbb{R}^{n}$ we refer to $[6]$.

With the above notations, we summarize the assumptions of Theorem (1) assuming that there exists $K>1$ such that: $\left|\nabla u^{0}\right| \leq K ;\left|H_{u^{0}}\right| \leq K$; the map $y \mapsto \nu(y)$ is Lipschitzean of constant $K$. Moreover, $d_{H}\left(T\left(y^{1}\right), T\left(y^{2}\right)\right) \leq K\left|y^{2}-y^{1}\right|$. In addition, there exists $M \geq 1$ such that for $x \in \Omega,|u(x)| \leq M,\left|u^{0}(x)\right| \leq M$. 
In what follows, a constant $h$ will be chosen; apart from further conditions, we shall always assume that $h>3 K$.

Definition 1. For $x \in \Omega$, set

$$
w_{+}^{h}(x)=\min \left\{u^{0}(z)+h|z-x|: z \in \partial \Omega\right\}
$$

and

$$
w_{-}^{h}(x)=\max \left\{u^{0}(z)-h|z-x|: z \in \partial \Omega\right\}
$$

Finally, set

$$
M^{h}(x)= \begin{cases}w_{+}^{h}(x) & \text { when } u(x)>w_{+}^{h}(x) \\ u(x) & \text { when } w_{-}^{h}(x) \leq u(x) \leq w_{+}^{h}(x) \\ w_{-}^{h}(x) & \text { when } u(x)<w_{-}^{h}(x)\end{cases}
$$

The following Lemmas will be essential to the proof of Theorem 1 . They will be used to smooth the approximating function $M^{h}$.

Lemma 1. Let $\Omega$ and $u^{0}$ be as in Theorem 1. Let $y=y(x)$ be a point where $w_{+}^{h}(x)=u^{0}(y(x))+h|y(x)-x|$. Then

i) $|y-x| \leq \frac{h+K}{h-K} d(x) \leq 2 d(x)$ and $\left|w_{+}^{h}(x)-u^{0}(y(x))\right| \leq[K+h] d(x)$ and

ii) (uniqueness) there exist $h^{*}$ and $d^{*}: h \geq h^{*}$ and $d(x) \leq d^{*}$ imply that $y=y(x)$ is uniquely defined and we have

$$
|y-x|=\frac{w_{+}^{h}(x)-u^{0}(y)}{h} .
$$

The same inequalities hold for $w_{-}^{h}$, provided that in ii) we read $|y-x|=\frac{u^{0}(y)-w_{-}^{h}(x)}{h}$.

Proof. We shall prove the inequalities for $w_{+}^{h}$. Ad i). Let $y^{*} \in \partial \Omega$ be such that $\left|y^{*}-x\right|=d(x)$. From the definition of $w_{+}^{h}$ we have that $u^{0}\left(y^{*}\right)+h d(x) \geq u^{0}(y)+$ $h|y-x|$, hence $h|y-x| \leq h d(x)+\left|u^{0}\left(y^{*}\right)-u^{0}(y)\right| \leq h d(x)+K\left|y^{*}-y\right| \leq h d(x)+$ $K[|y-x|+d(x)]$, so that

$$
|y-x| \leq\left(\frac{h+K}{h-K}\right) d(x)
$$

again from $u^{0}\left(y^{*}\right)+h d(x) \geq w_{+}^{h}(x)$ we infer

$$
\left|w_{+}^{h}(x)-u^{0}(x)\right| \leq\left|u^{0}\left(y^{*}\right)-u^{0}(x)\right|+h d(x) \leq K\left|y^{*}-x\right|+h d(x)=[K+h] d(x)
$$

thus proving i).

Ad ii). Whenever the minimum is attained at a point $y$, since $y$ is a constrained minimum point, we must have

$$
\nabla u^{0}(y)+h \frac{y-x}{|y-x|}=\nabla\left(u^{0}(y)+h|y-x|\right)=\lambda \nu(y)
$$

so that, for any $\tau$ in $T(y)$,

$$
\left\langle\nabla u^{0}(y), \tau\right\rangle=-h\left\langle\frac{y-x}{|y-x|}, \tau\right\rangle .
$$

Assume that $y^{1}$ and $y^{2}$ are points where the minimum is attained; set $r=\mid x-$ $y^{2}|-| x-y^{1} \mid$, so that $|r| \leq\left|y^{2}-y^{1}\right|$.

For any $\tau^{i} \in T\left(y^{i}\right)$, from (5) we infer

$$
0=\left\langle x-y^{2}+\left|x-y^{2}\right| \frac{\nabla u^{0}\left(y^{2}\right)}{h}, \tau^{2}\right\rangle=\left\langle x-y^{1}+\left|x-y^{1}\right| \frac{\nabla u^{0}\left(y^{1}\right)}{h}, \tau^{1}\right\rangle
$$


so that

$$
\begin{gathered}
\left\langle x-y^{2}+\left|x-y^{2}\right| \frac{\nabla u^{0}\left(y^{2}\right)}{h}, \tau^{1}\right\rangle-\left\langle x-y^{1}+\left|x-y^{1}\right| \frac{\nabla u^{0}\left(y^{1}\right)}{h}, \tau^{1}\right\rangle= \\
\left\langle x-y^{2}+\left|x-y^{2}\right| \frac{\nabla u^{0}\left(y^{2}\right)}{h}, \tau^{1}-\tau^{2}\right\rangle .
\end{gathered}
$$

There exists $\eta^{*}$ such that: for any $y^{2}$ with $\left|y^{2}-y^{1}\right| \leq \eta^{*}$ there is $\tau \in T\left(y^{1}\right)$ (with $\tau$ depending on $y^{2}$ ) such that

We have

$$
\left\langle\frac{y^{1}-y^{2}}{\left|y^{1}-y^{2}\right|}, \tau\right\rangle \geq \frac{1}{2}
$$

$$
\begin{gathered}
\left\langle x-y^{2}+\left|x-y^{2}\right| \frac{\nabla u^{0}\left(y^{2}\right)}{h}, \tau\right\rangle-\left\langle x-y^{1}+\left|x-y^{1}\right| \frac{\nabla u^{0}\left(y^{1}\right)}{h}, \tau\right\rangle \\
=\left\langle y^{1}-y^{2}+r \frac{\nabla u^{0}\left(y^{2}\right)}{h}, \tau\right\rangle-\left|x-y^{1}\right|\left\langle\frac{\left.\nabla u^{0}\left(y^{1}\right)-\nabla u^{0}\left(y^{2}\right)\right)}{h}, \tau\right\rangle \\
=\left|y^{1}-y^{2}\right|\left\langle\frac{y^{1}-y^{2}}{\left|y^{1}-y^{2}\right|}, \tau\right\rangle+r\left\langle\frac{\nabla u^{0}\left(y^{2}\right)}{h}, \tau\right\rangle-\left|x-y^{1}\right|\left\langle\frac{\left.\nabla u^{0}\left(y^{1}\right)-\nabla u^{0}\left(y^{2}\right)\right)}{h}, \tau\right\rangle .
\end{gathered}
$$

Set $d^{1}=\min \left\{\frac{\eta^{*}}{4}, 1\right\}$, so that $d(x) \leq d^{1}$ implies $\left|y^{1}-y^{2}\right| \leq \eta^{*}$ and, from equation (6), we obtain, for any $\tau^{1} \in T\left(y^{1}\right)$,

$$
\left\langle x-y^{2}+\left|x-y^{2}\right| \frac{\nabla u^{0}\left(y^{2}\right)}{h}, \tau^{1}-\tau^{2}\right\rangle \geq \frac{1}{2}\left|y^{1}-y^{2}\right|-3\left|y^{2}-y^{1}\right| \frac{K}{h} .
$$

Consider the left hand side for $\tau^{1}=\tau$; choose $\tau^{2} \in T^{1}\left(y^{2}\right)$ so that $\left|\tau^{2}-\tau\right| \leq$ $K\left|y^{1}-y^{2}\right|$; we obtain

$$
2 d(x)\left(1+\frac{K}{h}\right) K\left|y^{1}-y^{2}\right| \geq \frac{1}{2}\left|y^{1}-y^{2}\right|-3\left|y^{2}-y^{1}\right| \frac{K}{h}
$$

choosing $h=12 K$ and $d^{*}=\min \left\{d^{1}, \frac{1}{20 K}\right\}$, the previous inequality implies $\mid y^{2}-$ $y^{1} \mid=0$.

It is easy to check that $\nabla w_{+}^{h}$ is constant of norm $h$ along the line segment joining $y$ to $x$ and is directed in the direction from $y$ to $x$; hence we have the identity

$$
|y-x|=\frac{w_{+}^{h}(x)-u^{0}(y)}{h} \text {. }
$$

Lemma 2. Let $v \in W^{1,1}(\Omega)$ be such that $|v(x)| \leq M$ a.e. on $\Omega$ and, on $\Omega \backslash \Omega_{\delta}$, define the function

$$
\tilde{v}(x)=\frac{1}{|B(x, \delta)|} \int_{B(x, \delta)} v(z) d z .
$$

Then: i) $\tilde{v}$ is Lipschitzean of constant $N M \frac{1}{\delta}$ and, ii) $\tilde{v}$ is a.e. differentiable and, at a point $x$ of differentiability, we have $\nabla \tilde{v}(x)=\frac{1}{\omega_{N} \delta^{N}} \int_{B(0, \delta)} \nabla v(x-z) d z$.

Proof. Ad i).

$$
\begin{gathered}
\left|\tilde{v}\left(x^{2}\right)-\tilde{v}\left(x^{1}\right)\right|=\left|\frac{1}{\left|B\left(x^{2}, \delta\right)\right|} \int_{B\left(x^{2}, \delta\right)} v(z) d z-\frac{1}{\left|B\left(x^{1}, \delta\right)\right|} \int_{B\left(x^{1}, \delta\right)} v(z) d z\right| \\
\leq \frac{1}{\omega_{N} \delta^{N}} M\left|B\left(x^{1}, \delta\right) \triangle B\left(x^{2}, \delta\right)\right|
\end{gathered}
$$


and $\left|B\left(x^{1}, \delta\right) \triangle B\left(x^{2}, \delta\right)\right| \leq 2 \omega_{N} \delta^{N} \leq \omega_{N} \delta^{N-1}\left|x^{1}-x^{2}\right|$ when $\left|x^{1}-x^{2}\right| \geq 2 \delta$, while, when $\left|x^{1}-x^{2}\right|<2 \delta,\left|B\left(x^{1}, \delta\right) \triangle B\left(x^{2}, \delta\right)\right| \leq \omega_{N}\left[\left(\delta+\left|x^{1}-x^{2}\right|\right)^{N}-\delta^{N}\right] \leq$ $N \omega_{N} \delta^{N-1}\left|x^{1}-x^{2}\right|$ so that, in either case,

$$
\left|\tilde{v}\left(x^{2}\right)-\tilde{v}\left(x^{1}\right)\right| \leq N M \frac{1}{\delta}\left|x^{1}-x^{2}\right| .
$$

Ad ii). From i) we have that there exists $\Omega_{\delta}^{*} \subset \Omega_{\delta}$ of full measure, such that $\tilde{v}$ is differentiable on $\Omega_{\delta}^{*}$. Hence, for $x \in \Omega_{\delta}^{*}$, there exists a vector $\nabla \tilde{v}(x)$ and a function $\varepsilon(h), \varepsilon(h) \rightarrow 0$ as $h \rightarrow 0$, such that, for every $h$ sufficiently small, we have

$$
\tilde{v}(x+h)-\tilde{v}(x)=\langle\nabla \tilde{v}(x), h\rangle+|h| \varepsilon(h) .
$$

Consider one coordinate direction $e_{i}$. On almost every line parallel to $e_{i}$, the map $t \mapsto v\left(x+t e_{i}\right)$ is absolutely continuous; there exists $\Omega_{\delta}^{i}$ of full measure such that $x \in \Omega_{\delta}^{i}$ and $t$ small imply

$$
\begin{aligned}
\tilde{v}\left(x+t e_{i}\right)-\tilde{v}(x)= & \frac{1}{\omega_{N} \delta^{N}} \int_{B(0, \delta)} v\left(x-z+t e_{i}\right)-v(x-z) d z \\
= & \frac{1}{\omega_{N} \delta^{N}} \int_{B(0, \delta)}\left[\int_{0}^{1}\left\langle\nabla u\left(x-z+s t e_{i}\right), t e_{i}\right\rangle d s\right] d z \\
= & \frac{1}{\omega_{N} \delta^{N}}\left[\int_{B(0, \delta)}\left\langle\nabla v(x-z), t e_{i}\right\rangle d z\right. \\
& \left.+\int_{B(0, \delta)}\left[\int_{0}^{1}\left\langle\nabla u\left(x-z+s t e_{i}\right)-\nabla v(x-z), t e_{i}\right\rangle d s\right] d z\right] \\
= & \left\langle\frac{1}{\omega_{N} \delta^{N}} \int_{B(0, \delta)} \nabla v(x-z) d z, t e_{i}\right\rangle+r_{i}(t)
\end{aligned}
$$

and

$$
\begin{aligned}
r_{i}(t)= & \frac{1}{\omega_{N} \delta^{N}} \int_{0}^{1}\left[\int_{\left[B(0, \delta)-s t e_{i}\right] \backslash B(0, \delta)}\left\langle\nabla v(x-z), t e_{i}\right\rangle d z\right. \\
& \left.-\int_{B(0, \delta) \backslash\left[B(0, \delta)-s t e_{i}\right]}\left\langle\nabla v(x-z), t e_{i}\right\rangle d z\right] d s
\end{aligned}
$$

so that $\frac{r_{i}(t)}{|t|} \rightarrow 0$. Hence, for $x \in \Omega_{\delta}^{*} \cap\left[\cap_{i} \Omega_{\delta}^{i}\right]$, we have

$$
\nabla \tilde{v}(x)=\frac{1}{\omega_{N} \delta^{N}} \int_{B(0, \delta)} \nabla v(x-z) d z .
$$

Lemma 3. Assume that either i) $g$ is measurable and such that $|g(x)| \leq D d(x)$ or, ii), that $g$ is Lipschitzean with Lipschitz constant $D$. Then, there exists $D^{*}$ such that the function

is Lipschitzean of constant $D^{*}$.

$$
\tilde{g}(x)=\frac{1}{|B(x, d(x))|} \int_{B(x, d(x))} g(z) d z
$$

Proof. Fix $x^{1}$ and $x^{2}$, let $d\left(x^{2}\right) \geq d\left(x^{1}\right)$, let $y^{1}$ and $y^{2}$ in $\partial \Omega$ be the nearest points to $x^{1}$ and $x^{2}$. From $\left|x^{2}-y^{2}\right| \leq\left|x^{2}-y^{1}\right| \leq\left|x^{2}-x^{1}\right|+\left|x^{1}-y^{1}\right|$, we obtain

$$
\left|x^{2}-x^{1}\right| \geq d\left(x^{2}\right)-d\left(x^{1}\right) .
$$


On the segment $\left[y^{2}, x^{2}\right]$, let $x^{2 *}$ be such that $d\left(x^{2 *}\right)=\left|y^{2}-x^{2 *}\right|=d\left(x^{1}\right)$. We have (9) $\left|x^{1}-x^{2 *}\right| \leq\left|x^{1}-x^{2}\right|+\left|x^{2}-x^{2 *}\right|=\left|x^{1}-x^{2}\right|+\left(d\left(x^{2}\right)-d\left(x^{1}\right)\right) \leq 2\left|x^{1}-x^{2}\right|$.

Ad i). We have

$$
\begin{aligned}
& \left|\frac{1}{\left|B\left(x^{2}, d\left(x^{2}\right)\right)\right|} \int_{B\left(x^{2}, d\left(x^{2}\right)\right)} g(z) d z-\frac{1}{\left|B\left(x^{1}, d\left(x^{1}\right)\right)\right|} \int_{B\left(x^{1}, d\left(x^{1}\right)\right)} g(z) d z\right| \\
& \quad \leq \frac{1}{\left|B\left(x^{2}, d\left(x^{2}\right)\right)\right|}\left|\int_{B\left(x^{2}, d\left(x^{2}\right)\right)} g(z) d z-\int_{B\left(x^{1}, d\left(x^{1}\right)\right)} g(z) d z\right| \\
& \quad+\int_{B\left(x^{1}, d\left(x^{1}\right)\right)}|g(z)| d z\left|\frac{1}{\left|B\left(x^{2}, d\left(x^{2}\right)\right)\right|}-\frac{1}{\left|B\left(x^{1}, d\left(x^{1}\right)\right)\right|}\right|=\alpha+\beta
\end{aligned}
$$

Consider $\alpha$.

$$
\begin{gathered}
\alpha \leq \frac{1}{\left|B\left(x^{2}, d\left(x^{2}\right)\right)\right|}\left\{\left|\int_{B\left(x^{2}, d\left(x^{2}\right)\right)} g(z) d z-\int_{B\left(x^{2 *}, d\left(x^{2 *}\right)\right)} g(z) d z\right|\right. \\
\left.+\left|\int_{B\left(x^{2 *}, d\left(x^{2 *}\right)\right)} g(z) d z-\int_{B\left(x^{1}, d\left(x^{1}\right)\right)} g(z) d z\right|\right\}=\frac{1}{\left|B\left(x^{2}, d\left(x^{2}\right)\right)\right|}\left\{\alpha_{1}+\alpha_{2}\right\} .
\end{gathered}
$$

Since $B\left(x^{2 *}, d\left(x^{2 *}\right)\right) \subset B\left(x^{2}, d\left(x^{2}\right)\right)$, we have

$$
\begin{aligned}
& \alpha_{1}=\left|\int_{B\left(x^{2}, d\left(x^{2}\right)\right) \backslash B\left(x^{2 *}, d\left(x^{2 *}\right)\right)} g(z) d z\right| \leq \omega_{N}\left[\left(d\left(x^{2}\right)\right)^{N}-\left(d\left(x^{2 *}\right)\right)^{N}\right] \cdot 2 D d\left(x^{2}\right) \\
& \leq 2 D \omega_{N} P_{N}\left(d\left(x^{2}\right)\right)^{N}\left(d\left(x^{2}\right)-d\left(x^{2 *}\right)\right)=2 D \omega_{N} P_{N}\left(d\left(x^{2}\right)\right)^{N}\left(d\left(x^{2}\right)-d\left(x^{1}\right)\right) .
\end{aligned}
$$

Also,

$$
\alpha_{2} \leq \int_{B\left(x^{2 *}, d\left(x^{1}\right)\right) \triangle B\left(x^{1}, d\left(x^{1}\right)\right)}|g(z) d z| \leq 2 D d\left(x^{1}\right)\left|B\left(x^{2 *}, d\left(x^{1}\right)\right) \triangle B\left(x^{1}, d\left(x^{1}\right)\right)\right|,
$$

and we have: when $2 d\left(x^{1}\right) \leq\left|x^{1}-x^{2 *}\right|$, it follows $\left|B\left(x^{2 *}, d\left(x^{1}\right)\right) \triangle B\left(x^{1}, d\left(x^{1}\right)\right)\right|=$ $2 \omega_{N}\left(d\left(x^{1}\right)\right)^{N} \leq \omega_{N}\left(d\left(x^{1}\right)\right)^{N-1}\left|x^{1}-x^{2 *}\right|$; when $2 d\left(x^{1}\right)>\left|x^{1}-x^{2 *}\right|$,

$$
\begin{aligned}
& \left.\left|B\left(x^{2 *}, d\left(x^{1}\right)\right) \triangle B\left(x^{1}, d\left(x^{1}\right)\right)\right| \leq \omega_{N}\left[\left(d\left(x^{1}\right)+\left|x^{1}-x^{2 *}\right|\right)^{N}-d\left(x^{1}\right)\right)^{N}\right] \\
\leq & \omega_{N}\left|x^{1}-x^{2 *}\right| P_{N}\left(d\left(x^{1}\right)+\left|x^{1}-x^{2 *}\right|\right)^{N-1} \leq \omega_{N}\left|x^{1}-x^{2 *}\right| P_{N}\left(3 d\left(x^{1}\right)\right)^{N-1} .
\end{aligned}
$$

In either case,

(10) $\quad\left|B\left(x^{2 *}, d\left(x^{1}\right)\right) \triangle B\left(x^{1}, d\left(x^{1}\right)\right)\right| \leq \omega_{N}\left|x^{1}-x^{2 *}\right| 3^{N-1} P_{N}\left(d\left(x^{1}\right)\right)^{N-1}$.

Hence, $\alpha_{2} \leq 2 \cdot 3^{N-1} D \omega_{N} P_{N}\left(d\left(x^{1}\right)\right)^{N}\left|x^{1}-x^{2 *}\right|$, so that

$$
\alpha \leq 2 D P_{N}\left[\left(d\left(x^{2}\right)-d\left(x^{1}\right)\right)+3^{N-1}\left|x^{1}-x^{2 *}\right|\right] .
$$

From (8) and (9) we obtain

$$
\alpha \leq 2 D P_{N}\left(1+2 \cdot 3^{N-1}\right)\left|x^{1}-x^{2}\right| .
$$

Consider $\beta$. We have $\int_{B\left(x^{1}, d\left(x^{1}\right)\right)}|g(z)| d z \leq \omega_{N}\left(d\left(x^{1}\right)\right)^{N} \cdot 2 D d\left(x^{1}\right)$ and

$$
\begin{gathered}
\left|\frac{1}{\left|B\left(x^{2}, d\left(x^{2}\right)\right)\right|}-\frac{1}{\left|B\left(x^{1}, d\left(x^{1}\right)\right)\right|}\right|=\frac{\left|B\left(x^{2}, d\left(x^{2}\right)\right)\right|-\left|B\left(x^{1}, d\left(x^{1}\right)\right)\right|}{\left|B\left(x^{1}, d\left(x^{1}\right)\right)\right|\left|B\left(x^{2}, d\left(x^{2}\right)\right)\right|} \\
=\frac{1}{\omega_{N}} \frac{\left(d\left(x^{2}\right)\right)^{N}-\left(d\left(x^{1}\right)\right)^{N}}{\left(d\left(x^{2}\right)\right)^{N}\left(d\left(x^{1}\right)\right)^{N}} \leq \frac{P_{N}}{\omega_{N}} \frac{\left(d\left(x^{2}\right)-d\left(x^{1}\right)\right)}{d\left(x^{2}\right)\left(d\left(x^{1}\right)\right)^{N}}
\end{gathered}
$$


so that

$$
\beta \leq 2 D P_{N}\left(d\left(x^{2}\right)-d\left(x^{1}\right)\right) \leq 2 D P_{N}\left|x^{2}-x^{1}\right| .
$$

We have obtained

$$
\left|\tilde{g}\left(x^{2}\right)-\tilde{g}\left(x^{1}\right)\right| \leq 2 D P_{N}\left(2+2 \cdot 3^{N}\right)\left|x^{2}-x^{1}\right| .
$$

Ad ii).

$$
\begin{aligned}
\left|\tilde{g}\left(x^{2}\right)-\tilde{g}\left(x^{1}\right)\right| & =\mid g\left(x^{2}\right)-g\left(x^{1}\right)+\frac{1}{\left|B\left(x^{2}, d\left(x^{2}\right)\right)\right|} \int_{B\left(x^{2}, d\left(x^{2}\right)\right)}\left(g(z)-g\left(x^{2}\right)\right) d z \\
& -\frac{1}{\left|B\left(x^{1}, d\left(x^{1}\right)\right)\right|} \int_{B\left(x^{1}, d\left(x^{1}\right)\right)}\left(g(z)-g\left(x^{1}\right)\right) d z \mid
\end{aligned}
$$

a) When $\left|x^{2}-x^{1}\right| \geq d\left(x^{2}\right)+d\left(x^{1}\right)$

$$
\begin{aligned}
& \mid \frac{1}{\left|B\left(x^{2}, d\left(x^{2}\right)\right)\right|} \int_{B\left(x^{2}, d\left(x^{2}\right)\right)}\left(g(z)-g\left(x^{2}\right)\right) d z \\
& \quad-\frac{1}{\left|B\left(x^{1}, d\left(x^{1}\right)\right)\right|} \int_{B\left(x^{1}, d\left(x^{1}\right)\right)}\left(g(z)-g\left(x^{1}\right)\right) d z \mid \\
& \leq D d\left(x^{2}\right)+D d\left(x^{1}\right) \leq D\left|x^{2}-x^{1}\right| .
\end{aligned}
$$

b) Let $\left|x^{2}-x^{1}\right| \leq d\left(x^{2}\right)+d\left(x^{1}\right)$. We have

$$
\begin{gathered}
\leq\left|\tilde{g}\left(x^{2}\right)-\tilde{g}\left(x^{1}\right)\right| \\
\leq\left|g\left(x^{2}\right)-g\left(x^{1}\right)\right|+\left|\frac{1}{\omega_{N}\left(d\left(x^{2}\right)\right)^{N}} \int_{B\left(x^{2}, d\left(x^{2}\right)\right) \backslash B\left(x^{1}, d\left(x^{1}\right)\right)}\left(g(z)-g\left(x^{2}\right)\right) d z\right| \\
+\left|\frac{1}{\omega_{N}\left(d\left(x^{1}\right)\right)^{N}} \int_{B\left(x^{1}, d\left(x^{1}\right)\right) \backslash B\left(x^{2}, d\left(x^{2}\right)\right)}\left(g(z)-g\left(x^{1}\right)\right) d z\right| \\
+\left|\int_{B\left(x^{1}, d\left(x^{1}\right)\right) \cap B\left(x^{2}, d\left(x^{2}\right)\right)}\left[\frac{1}{\omega_{N}\left(d\left(x^{2}\right)\right)^{N}}\left(g(z)-g\left(x^{2}\right)\right)-\frac{1}{\omega_{N}\left(d\left(x^{1}\right)\right)^{N}}\left(g(z)-g\left(x^{1}\right)\right)\right] d z\right| \\
=\left|g\left(x^{2}\right)-g\left(x^{1}\right)\right|+\alpha+\beta+\gamma .
\end{gathered}
$$

We have

$$
|\alpha| \leq \operatorname{Dd}\left(x^{2}\right) \frac{1}{\left(d\left(x^{2}\right)\right)^{N}}\left[\left(d\left(x^{2}\right)+\left|x^{2}-x^{1}\right|\right)^{N}-\left(d\left(x^{1}\right)\right)^{N}\right] ;
$$

since $d\left(x^{2}\right)-d\left(x^{1}\right) \leq\left|x^{2}-x^{1}\right| \leq 2 d\left(x^{2}\right)$, we obtain

$$
|\alpha| \leq \frac{D}{\left(d\left(x^{2}\right)\right)^{N-1}} 2\left|x^{2}-x^{1}\right| P_{N}\left(3 d\left(x^{2}\right)\right)^{N-1}=2 P_{N} 3^{N-1} D\left|x^{2}-x^{1}\right| .
$$

Consider $\beta$; we have

$$
|\beta| \leq \frac{D}{\omega_{N}\left(d\left(x^{1}\right)\right)^{N-1}}\left|B\left(x^{1}, d\left(x^{1}\right)\right) \backslash B\left(x^{2}, d\left(x^{2}\right)\right)\right| .
$$

Since $B\left(x^{1}, d\left(x^{2}\right)-\left|x^{2}-x^{1}\right|\right) \subset B\left(x^{2}, d\left(x^{2}\right)\right)$, we infer $B\left(x^{1}, d\left(x^{1}\right)\right) \backslash B\left(x^{2}, d\left(x^{2}\right)\right) \subset$ $B\left(x^{1}, d\left(x^{1}\right)\right) \backslash B\left(x^{1}, d\left(x^{2}\right)-\left|x^{2}-x^{1}\right|\right)$, hence

$$
\begin{gathered}
\left|B\left(x^{1}, d\left(x^{1}\right)\right) \backslash B\left(x^{2}, d\left(x^{2}\right)\right)\right| \leq \omega_{N}\left[\left(\left(d\left(x^{1}\right)\right)^{N}-\left(d\left(x^{2}\right)-\left|x^{2}-x^{1}\right|\right)^{N}\right]\right. \\
\leq \omega_{N}\left(d\left(x^{1}\right)-d\left(x^{2}\right)+\left|x^{2}-x^{1}\right|\right) P_{N}\left(2 d\left(x^{1}\right)\right)^{N-1} \leq \omega_{N}\left|x^{2}-x^{1}\right| P_{N}\left(2 d\left(x^{1}\right)\right)^{N-1}
\end{gathered}
$$
and

$$
|\beta| \leq D P_{N} 2^{N-1}\left|x^{2}-x^{1}\right|
$$


Consider $\gamma$; write the absolute value of the integrand as

$$
\begin{gathered}
\mid \frac{1}{\omega_{N}\left(d\left(x^{2}\right)\right)^{N}}\left(g(z)-g\left(x^{2}\right)\right)-\frac{1}{\omega_{N}\left(d\left(x^{2}\right)\right)^{N}}\left(g(z)-g\left(x^{1}\right)\right) \\
+\frac{1}{\omega_{N}\left(d\left(x^{2}\right)\right)^{N}}\left(g(z)-g\left(x^{1}\right)\right)-\frac{1}{\omega_{N}\left(d\left(x^{1}\right)\right)^{N}}\left(g(z)-g\left(x^{1}\right)\right) \mid \\
=\left|\frac{1}{\omega_{N}\left(d\left(x^{2}\right)\right)^{N}}\left(g\left(x^{1}\right)-g\left(x^{2}\right)\right)+\left(g(z)-g\left(x^{1}\right)\right)\left(\frac{1}{\omega_{N}\left(d\left(x^{2}\right)\right)^{N}}-\frac{1}{\omega_{N}\left(d\left(x^{1}\right)\right)^{N}}\right)\right|
\end{gathered}
$$

Since $\left|B\left(x^{1}, d\left(x^{1}\right)\right) \cap B\left(x^{2}, d\left(x^{2}\right)\right)\right| \leq \omega_{N}\left(d\left(x^{1}\right)\right)^{N}$, we obtain

$$
\begin{gathered}
\gamma \leq\left[\frac{D}{\omega_{N}\left(d\left(x^{2}\right)\right)^{N}}\left|x^{2}-x^{1}\right|+D d\left(x^{1}\right)\left(\frac{\left(d\left(x^{2}\right)\right)^{N}-\left(d\left(x^{1}\right)\right)^{N}}{\omega_{N}\left(d\left(x^{1}\right)\right)^{N}\left(d\left(x^{2}\right)\right)^{N}}\right)\right] \omega_{N}\left(d\left(x^{1}\right)\right)^{N} \\
\leq D\left|x^{2}-x^{1}\right|+D \frac{d\left(x^{1}\right)}{d\left(x^{2}\right)} P_{N}\left(d\left(x^{2}\right)-d\left(x^{1}\right)\right) \leq D\left(1+P_{N}\right)\left|x^{2}-x^{1}\right| .
\end{gathered}
$$

\section{Differentiability Results}

Let $P \in \partial \Omega$; we choose as coordinate system (depending on $P$ ) the one that has the origin in $P$ and the $x_{N}$ axis in the direction of the normal to the inside of $\Omega$, so that, for $i<N$, the $x_{i}$ axis is on the tangent plane to $P$. On this system, $\partial \Omega$ is described locally by $x_{N}=\phi(\hat{x})$, with $\phi$ a smooth function such that $\phi(\hat{0})=\nabla \phi(\hat{0})=0$; given $\Phi \leq 1$, we shall call $B_{\Phi}(P)$ the maximal open ball centered at $\hat{0}$ in $\mathbb{R}^{N-1}$ such that, for $\hat{x} \in B_{\Phi}$, we have $|\nabla \phi(\hat{x})|<\Phi$.

Set

$$
\bar{\nu}=\left(\begin{array}{l}
-\phi_{x_{1}} \\
\cdot \\
\cdot \\
-\phi_{x_{N-1}} \\
1
\end{array}\right) ; \bar{\tau}_{1}=\left(\begin{array}{l}
1 \\
0 \\
\cdot \\
\cdot \\
\cdot \\
\phi_{x_{1}}
\end{array}\right) ; \ldots: \bar{\tau}_{N-1}=\left(\begin{array}{l}
0 \\
\cdot \\
\cdot \\
\cdot \\
1 \\
\phi_{x_{N-1}}
\end{array}\right)
$$

and

$$
\nu=\frac{\bar{\nu}}{|\bar{\nu}|} ; \tau_{i}=\frac{\bar{\tau}_{i}}{\left|\bar{\tau}_{i}\right|}
$$

Given a point $x \in \Omega$, as before we denote by $y(x)$ the point in $\partial \Omega$ where the minimum in (1) is attained. We shall consider the map $x \mapsto \hat{y} ; J(\hat{y})$ is the Jacobian of this map.

Lemma 4 (Differentiability lemma). For every $\eta$ there exist $\tilde{h}$ and $\tilde{\Phi}$ such that $h \geq \tilde{h}$ and $\Phi \leq \tilde{\Phi}$ imply that the map $x \mapsto \hat{y}$ is well defined and differentiable on $\Omega_{\frac{3 M}{h}}$, and we have

$$
\frac{1-\eta}{1+\eta} \leq J(\hat{y}) \leq \frac{\sqrt{1+\eta^{2}}}{(1-\eta)} .
$$

Being the case $N=2$ substantially simpler than the general case, we present it separately. In the Proof of this Lemma we shall consider partial derivatives evaluated at different points; it will be convenient to set $f_{j}^{\prime}$ to denote the partial derivative of the (scalar-valued) function $f$ with respect to its $j$-th variable. 
Proof. The case $N=2$. a) We first claim that the map $\nabla w_{+}^{h}$ is a known function when computed at a generic point $\left(y_{1}, \phi\left(y_{1}\right)\right) \in \partial \Omega$. In fact, from $u^{0}\left(y_{1}, \phi\left(y_{1}\right)\right) \equiv$ $w_{+}^{h}\left(y_{1}, \phi\left(y_{1}\right)\right)$ we obtain

$$
\frac{d}{d y_{1}} u^{0}\left(y_{1}, \phi\left(y_{1}\right)\right)=\left\langle\nabla u^{0}, \bar{\tau}\right\rangle=\frac{d}{d y_{1}} w_{+}^{h}\left(y_{1}, \phi\left(y_{1}\right)\right)=\left\langle\nabla w_{+}^{h}, \bar{\tau}\right\rangle
$$

so that

$$
\left\langle\nabla w_{+}^{h}\left(y_{1}, \phi\left(y_{1}\right)\right), \tau\right\rangle \equiv\left\langle\nabla u^{0}\left(y_{1}, \phi\left(y_{1}\right)\right), \tau\right\rangle ;
$$

since the norm of $\nabla w_{+}^{h}$ is $h$, we also have

$$
\left\langle\nabla w_{+}^{h}\left(y_{1}, \phi\left(y_{1}\right)\right), \nu\right\rangle=\sqrt{h^{2}-\left\langle\nabla u^{0}\left(y_{1}, \phi\left(y_{1}\right)\right), \tau\right\rangle^{2}} .
$$

Let $e_{i}$ be the coordinate directions; writing

$$
e_{1}=\left\langle\tau, e_{1}\right\rangle \tau+\left\langle\nu, e_{1}\right\rangle \nu ; \quad e_{2}=\left\langle\tau, e_{2}\right\rangle \tau+\left\langle\nu, e_{2}\right\rangle \nu
$$

we obtain the cartesian coordinates of $\nabla w_{+}^{h}$, i.e.,

$$
\begin{gathered}
\left(\begin{array}{c}
\left(w_{+}^{h}\right)_{1}^{\prime} \\
\left(w_{+}^{h}\right)_{2}^{\prime}
\end{array}\right)=\left(\begin{array}{c}
\left\langle\nabla w_{+}^{h}, e_{1}\right\rangle \\
\left\langle\nabla w_{+}^{h}, e_{2}\right\rangle
\end{array}\right)=\left(\begin{array}{c}
\left\langle\nabla w_{+}^{h}, \tau\right\rangle\left\langle\tau, e_{1}\right\rangle+\left\langle\nabla w_{+}^{h}, \nu\right\rangle\left\langle\nu, e_{1}\right\rangle \\
\left\langle\nabla w_{+}^{h}, \tau\right\rangle\left\langle\tau, e_{2}\right\rangle+\left\langle\nabla w_{+}^{h}, \nu\right\rangle\left\langle\nu, e_{2}\right\rangle
\end{array}\right) \\
=\left(\begin{array}{c}
\left\langle\nabla u^{0}, \tau\right\rangle\left\langle\tau, e_{1}\right\rangle+\sqrt{h^{2}-\left\langle\nabla u^{0}, \tau\right\rangle^{2}}\left\langle\nu, e_{1}\right\rangle \\
\left\langle\nabla u^{0}, \tau\right\rangle\left\langle\tau, e_{2}\right\rangle+\sqrt{h^{2}-\left\langle\nabla u^{0}, \tau\right\rangle^{2}}\left\langle\nu, e_{2}\right\rangle
\end{array}\right)
\end{gathered}
$$

In particular,

$$
\left.\left(w_{+}^{h}\right)_{1}^{\prime}\left(y_{1}, \phi\left(y_{1}\right)\right) \equiv\left[\left\langle\nabla u^{0}, \tau\right\rangle\left\langle\tau, e_{1}\right\rangle+\sqrt{h^{2}-\left\langle\nabla u^{0}, \tau\right\rangle^{2}}\left\langle\nu, e_{1}\right\rangle\right]\right|_{\left(y_{1}, \phi\left(y_{1}\right)\right)}
$$

b) Consider $h^{*}$ and $d^{*}$ defined in Lemma 1 . We can assume that $h^{*} \geq \frac{3 M}{d^{*}}$. For every $h \geq h^{*}$ and $d(x) \leq d^{*}$, the map (depending on $\left.h\right) x \mapsto y(x)=\left(y_{1}, \phi\left(y_{1}\right)\right)$ is well defined. We claim that $y_{1}$ is a differentiable function of $x$.

Recalling that $\nabla w_{+}^{h}$ is constant along the line segment joining $\left(x_{1}, x_{2}\right)$ and $\left(y_{1}, \phi\left(y_{1}\right)\right)$, we obtain the identity

$$
\nabla w_{+}^{h}\left(x_{1}, x_{2}\right)=\left(\begin{array}{c}
\left\langle\nabla u^{0}, \tau\right\rangle\left\langle\tau, e_{1}\right\rangle+\sqrt{h^{2}-\left\langle\nabla u^{0}, \tau\right\rangle^{2}}\left\langle\nu, e_{1}\right\rangle \\
\left\langle\nabla u^{0}, \tau\right\rangle\left\langle\tau, e_{2}\right\rangle+\sqrt{h^{2}-\left\langle\nabla u^{0}, \tau\right\rangle^{2}}\left\langle\nu, e_{2}\right\rangle
\end{array}\right)
$$

where the right hand side is computed at the point $\left(y_{1}(x), \phi\left(y_{1}(x)\right)\right)$.

The points $x$ and $y$ are related by the identity $x=y+|x-y| \frac{x-y}{|x-y|}$, i.e., from (7), by

$$
\left(\begin{array}{l}
x_{1} \\
x_{2}
\end{array}\right)=\left(\begin{array}{l}
y_{1}(x) \\
\phi\left(y_{1}(x)\right)
\end{array}\right)+\frac{\left(w_{+}^{h}(x)-u^{0}\left(y_{1}(x), \phi\left(y_{1}(x)\right)\right)\right)}{h} \frac{\nabla w_{+}^{h}\left(y_{1}(x), \phi\left(y_{1}(x)\right)\right.}{h} .
$$

in particular,

$$
x_{1} \equiv y_{1}+\frac{1}{h^{2}}\left(w_{+}^{h}\left(x_{1}, x_{2}\right)-u^{0}\left(y_{1}, \phi\left(y_{1}\right)\right)\left(w_{+}^{h}\right)_{1}^{\prime}\left(y_{1}, \phi\left(y_{1}\right)\right) ;\right.
$$

differentiating with respect to $x_{1}$ this identity, we have

and

$$
\begin{gathered}
1 \equiv\left(y_{1}\right)_{x_{1}}+\frac{1}{h^{2}}\left\{\left[\left(w_{+}^{h}\right)_{x_{1}}-\left(\left\langle\nabla u^{0}, \bar{\tau}\right\rangle \cdot\left(y_{1}\right)_{x_{1}}\right)\right]\left(w_{+}^{h}\right)_{1}^{\prime}\left(y_{1}, \phi\left(y_{1}\right)\right)\right. \\
\left.+\left(w_{+}^{h}-u^{0}\left(y_{1}, \phi\left(y_{1}\right)\right)\right)\left\langle\nabla\left(\left(w_{+}^{h}\right)_{1}^{\prime}\right), \bar{\tau}\right\rangle \cdot\left(y_{1}\right)_{x_{1}}\right\}
\end{gathered}
$$

$$
0 \equiv\left(y_{1}\right)_{x_{2}}+\frac{1}{h^{2}}\left\{\left[\left(w_{+}^{h}\right)_{x_{2}}-\left(\left\langle\nabla u^{0}, \bar{\tau}\right\rangle \cdot\left(y_{1}\right)_{x_{2}}\right)\right]\left(w_{+}^{h}\right)_{x_{1}}\left(y_{1}, \phi\left(y_{1}\right)\right)\right.
$$




$$
\left.+\left(w_{+}^{h}-u^{0}\left(y_{1}, \phi\left(y_{1}\right)\right)\right)\left\langle\nabla\left(\left(w_{+}^{h}\right)_{1}^{\prime}\right), \bar{\tau}\right\rangle \cdot\left(y_{1}\right)_{x_{2}}\right\} .
$$

From (16), we have $\left(w_{+}^{h}\right)_{i}^{\prime}\left(y_{1}, \phi\left(y_{1}\right)\right)=\left(w_{+}^{h}\right)_{i}^{\prime}\left(x_{1}, x_{2}\right)$ and we obtain

$$
\left(y_{1}\right)_{x_{1}}=\frac{1-\frac{1}{h^{2}}\left(\left(w_{+}^{h}\right)_{1}^{\prime}\right)^{2}}{1-\frac{1}{h^{2}}\left[\left\langle\nabla u^{0}, \bar{\tau}\right\rangle\left(w_{+}^{h}\right)_{1}^{\prime}-\left(w_{+}^{h}-u^{0}\left(y_{1}, \phi\left(y_{1}\right)\right)\right)\left\langle\nabla\left(\left(w_{+}^{h}\right)_{1}^{\prime}\right), \bar{\tau}\right\rangle\right]}
$$

and

$$
\left(y_{1}\right)_{x_{2}}=\frac{-\frac{1}{h^{2}}\left(w_{+}^{h}\right)_{1}^{\prime}\left(w_{+}^{h}\right)_{2}^{\prime}}{1-\frac{1}{h^{2}}\left[\left\langle\nabla u^{0}, \bar{\tau}\right\rangle\left(w_{+}^{h}\right)_{1}^{\prime}-\left(w_{+}^{h}-u^{0}\left(y_{1}, \phi\left(y_{1}\right)\right)\right)\left\langle\nabla\left(\left(w_{+}^{h}\right)_{1}^{\prime}\right), \bar{\tau}\right\rangle\right]} .
$$

c) We wish to estimate the Jacobian of the map $x \mapsto y_{1}$. Differentiating (15),

$$
\begin{aligned}
& \frac{d}{d y_{1}}\left(w_{+}^{h}\right)_{1}^{\prime}\left(y_{1}, \phi\left(y_{1}\right)\right)=\left\langle\nabla\left(\left(w_{+}^{h}\right)_{1}^{\prime}\right), \bar{\tau}\right\rangle=\left(\left\langle\nabla u^{0}, \tau\right\rangle\right)_{y_{1}}\left\langle\tau, e_{1}\right\rangle+\left\langle\nabla u^{0}, \tau\right\rangle\left(\left\langle\tau, e_{1}\right\rangle\right)_{y_{1}} \\
& -\frac{\left\langle\nabla u^{0}, \tau\right\rangle\left(\left\langle\nabla u^{0}, \tau\right\rangle\right)_{y_{1}}}{\sqrt{h^{2}-\left\langle\nabla u^{0}, \tau\right\rangle^{2}}}\left\langle\nu, e_{1}\right\rangle+\sqrt{h^{2}-\left\langle\nabla u^{0}, \tau\right\rangle^{2}}\left(\left\langle\nu, e_{1}\right\rangle\right)_{y_{1}}=A+B+C+D ;
\end{aligned}
$$

also

and

$$
\left(\frac{d}{d y_{1}}\left\langle\nabla u^{0}, \tau\right\rangle\right) \frac{1}{\sqrt{1+\left(\phi^{\prime}\right)^{2}}}=\tau^{T} H_{u^{0}} \tau+\frac{\phi^{\prime \prime}}{\left(1+\left(\phi^{\prime}\right)^{2}\right)^{\frac{3}{2}}}\left\langle\nabla u^{0}, \nu\right\rangle
$$

$$
\left(\left\langle\tau, e_{1}\right\rangle\right)_{y_{1}}=-\frac{\phi^{\prime} \phi^{\prime \prime}}{\left(1+\left(\phi^{\prime}\right)^{2}\right)^{\frac{3}{2}}} ; \quad\left(\left\langle\nu, e_{1}\right\rangle\right)_{y_{1}}=-\frac{\phi^{\prime \prime}}{\left(1+\left(\phi^{\prime}\right)^{2}\right)^{\frac{3}{2}}} .
$$

We have $\left|\left(w_{+}^{h}\right)_{1}^{\prime}\right| \leq h$ and $\left|\nabla u^{0}\right| \leq K ;\left|\left\langle\nabla u^{0}, \bar{\tau}\right\rangle\right| \leq K \sqrt{1+\left(\phi^{\prime}\left(y_{1}\right)\right)^{2}}$. Recalling that $\Phi<1$ and $h>3 K$,

$$
|A| \leq 2 K+K^{2} ; \quad|B| \leq K^{2} ; \quad|C| \leq \frac{2 K+K^{2}}{h} K \leq K^{2} ; \quad|D| \leq h K,
$$

so that

$$
\left|\frac{d}{d y_{1}}\left(w_{+}^{h}\right)_{1}^{\prime}\left(y_{1}, \phi\left(y_{1}\right)\right)\right| \leq K_{1}+K h .
$$

Recalling i) of Lemma 1 , on the set $\Omega_{\frac{3 M}{h}}$ we have $w_{+}^{h}(x)-u^{0}(y(x))=h|x-y(x)| \leq$ $h \cdot 2 \frac{3 M}{h}=6 M$, so that

$$
\left|\left(w_{+}^{h}(x)-u^{0}\left(y_{1}, \phi\left(y_{1}\right)\right)\right) \frac{1}{h^{2}}\left(\frac{d}{d y_{1}}\left(w_{+}^{h}\right)_{1}^{\prime}\right)\right| \leq 6 M \frac{1}{h^{2}}\left(K_{1}+K h\right) ;
$$

in addition,

$$
\left|\left\langle\nabla u^{0}, \bar{\tau}\right\rangle \frac{1}{h^{2}}\left(w_{+}^{h}\right)_{1}^{\prime}\right| \leq 2 K \frac{1}{h^{2}} h
$$

we have obtained that the denominator satisfies

$$
\begin{aligned}
1+\frac{2}{h^{2}} & {\left[3 M\left(K_{1}+2 K h\right)\right] } \\
& \geq 1-\frac{1}{h^{2}}\left[\left\langle\nabla u^{0}, \bar{\tau}\right\rangle\left(w_{+}^{h}\right)_{1}^{\prime}-\left(w_{+}^{h}-u^{0}\left(y_{1}, \phi\left(y_{1}\right)\right)\right)\left\langle\nabla\left(\left(w_{+}^{h}\right)_{1}^{\prime}\right), \bar{\tau}\right\rangle\right] \\
& \geq 1-\frac{2}{h^{2}}\left[3 M\left(K_{1}+2 K h\right)\right] .
\end{aligned}
$$

In addition, from (16), we have $\frac{1}{h}\left|w_{x_{1}}^{h}\right| \leq \frac{K}{h}+\Phi$ and $\left|\frac{1}{h^{2}} w_{x_{1}}^{h} w_{x_{2}}^{h}\right| \leq \frac{K}{h}+\Phi$ so that we can make either term arbitrarily small by choosing $\frac{1}{h}$ and $\Phi$ small.

d) Fix $\eta$. Fix $\tilde{h}$ so large and $\tilde{\Phi}$ so small that $h \geq \tilde{h}$ and $\Phi \leq \tilde{\Phi}$ imply:

$$
1-\eta \leq 1-\frac{1}{h^{2}}\left[\left\langle\nabla u^{0}, \bar{\tau}\right\rangle\left(w_{+}^{h}\right)_{1}^{\prime}-\left(w_{+}^{h}-u^{0}\left(y_{1}, \phi\left(y_{1}\right)\right)\right)\left\langle\nabla\left(\left(w_{+}^{h}\right)_{1}^{\prime}\right), \bar{\tau}\right\rangle\right] \leq 1+\eta ;
$$




$$
\frac{1}{h^{2}}\left(\left(w_{+}^{h}\right)_{1}^{\prime}\right)^{2} \leq \eta \text { and }\left|\frac{1}{h^{2}}\left(w_{+}^{h}\right)_{1}^{\prime}\left(w_{+}^{h}\right)_{2}^{\prime}\right| \leq \eta
$$

We obtain, for every $x \in \Omega_{\frac{3 M}{h}}$,

and

$$
\frac{1-\eta}{1+\eta} \leq\left(y_{1}\right)_{x_{1}} \leq \frac{1}{1-\eta} ; \quad 0 \leq\left|\left(y_{1}\right)_{x_{2}}\right| \leq \eta
$$

$$
\frac{1-\eta}{1+\eta} \leq J\left(\left(y_{1}\right)(x)\right)=\sqrt{\left(y_{1}\right)_{x_{1}}^{2}+\left(y_{1}\right)_{x_{2}}^{2}} \leq \frac{\sqrt{1+\eta^{2}}}{(1-\eta)} .
$$

Proof. The general case. a) Consider a generic point $(\hat{y}, \phi(\hat{y})) \in \partial \Omega$, so that $\tau_{i}=$ $\tau_{i}(\hat{y})$ and $\nu=\nu(\hat{y})$ : we claim that the map $\nabla w_{+}^{h}$ is known when computed at $(\hat{y}, \phi(\hat{y}))$. In fact, from $u^{0}(\hat{y}, \phi(\hat{y})) \equiv w_{+}^{h}(\hat{y}, \phi(\hat{y}))$, we obtain

so that

$$
\frac{d}{d y_{i}} u^{0}(\hat{y}, \phi(\hat{y}))=\left\langle\nabla u^{0}, \bar{\tau}_{i}\right\rangle=\frac{d}{d y_{i}} w_{+}^{h}(\hat{y}, \phi(\hat{y}))=\left\langle\nabla w_{+}^{h}, \bar{\tau}_{i}\right\rangle
$$

$$
\left\langle\nabla w_{+}^{h}(\hat{y}, \phi(\hat{y})), \tau_{i}\right\rangle=\left\langle\nabla u^{0}(\hat{y}, \phi(\hat{y})), \tau_{i}\right\rangle
$$

For a vector $v$ in $\mathbb{R}^{N}$, let $P(v)$ be the projection of $v$ on the tangent plane; write $v=\langle v, \nu\rangle \nu+\sum a_{i} \tau_{i}$, so that $\sum a_{i} \tau_{i}=P(v)$; we obtain, for the coefficients $a_{i}$, the system

$$
\left\langle v, \tau_{j}\right\rangle=\sum_{i} a_{i}\left\langle\tau_{i}, \tau_{j}\right\rangle
$$

In particular, for the vector $\nabla w_{+}^{h}$, we obtain

$$
\nabla w_{+}^{h}=\left\langle\nabla w_{+}^{h}, \nu\right\rangle \nu+\sum_{i=1, \ldots, N-1} a_{i} \tau_{i}
$$

and, from (19), (20) becomes

$$
\left\langle\nabla u^{0}, \tau_{j}\right\rangle=\sum a_{i}\left\langle\tau_{i}, \tau_{j}\right\rangle
$$

The coefficient matrix $T=\left(\left\langle\tau_{i}, \tau_{j}\right\rangle\right)$ of system $(22)$ converges to $\left(\delta_{i, j}\right)$ as $\Phi \rightarrow 0$; hence, for every $\Phi$ small, system (22) is solvable.

We also have

$$
h^{2}=\left|\nabla w_{+}^{h}\right|^{2}=\left\langle\nabla w_{+}^{h}, \nu\right\rangle^{2}+\left(P\left(\nabla w_{+}^{h}\right)\right)^{2}
$$

and we obtain

$$
\left\langle\nabla w_{+}^{h}, \nu\right\rangle=\sqrt{h^{2}-\left(\sum_{i=1}^{N-1} a_{i}^{2}+\sum_{i, l=1, \ldots, N-1 ; i \neq l} a_{i} a_{l}\left\langle\tau_{i}, \tau_{l}\right\rangle\right)}
$$

b) Equations (19) and (23) provide $\left\langle\nabla w_{+}^{h}, \tau_{i}\right\rangle$ and $\left\langle\nabla w_{+}^{h}, \nu\right\rangle$; in order to obtain the Cartesian coordinates of $\nabla w_{+}^{h}$, write, for $j=1, \ldots, N$,

$$
e_{j}=\left\langle e_{j}, \nu\right\rangle \nu+\sum_{i=1, \ldots, N-1} b_{i}^{j} \tau_{i}
$$

We have

$$
\left(w_{+}^{h}\right)_{j}^{\prime}=\left\langle\nabla w_{+}^{h}, e_{j}\right\rangle=\left\langle e_{j}, \nu\right\rangle\left\langle\nabla w_{+}^{h}, \nu\right\rangle+\sum_{i=1, \ldots, N-1} b_{i}^{j}\left\langle\nabla w_{+}^{h}, \tau_{i}\right\rangle
$$


hence

$$
\left\langle\nabla w_{+}^{h}, e_{j}\right\rangle(\hat{y}, \phi(\hat{y}))
$$

$$
\left.\equiv\left[\left\langle e_{j}, \nu\right\rangle \sqrt{h^{2}-\left(\sum_{i=1}^{N-1} a_{i}^{2}+\sum_{i, l=1, \ldots, N-1 ; i \neq l} a_{i} a_{l}\left\langle\tau_{i}, \tau_{l}\right\rangle\right)}+\sum_{i=1}^{N-1} b_{i}^{j}\left\langle\nabla u^{0}, \tau_{i}\right\rangle\right]\right|_{(\hat{y}, \phi(\hat{y}))} .
$$

c) We have the identity

(26) $\left(\begin{array}{l}\hat{x} \\ x_{N}\end{array}\right)=\left(\begin{array}{l}\hat{y}(x) \\ \phi(\hat{y}(x))\end{array}\right)+\frac{\left(w_{+}^{h}(x)-u^{0}(\hat{y}(x), \phi(\hat{y}(x)))\right)}{h} \frac{\nabla w_{+}^{h}(\hat{y}(x), \phi(\hat{y}(x))}{h}$.

Differentiate with respect to $x_{j}$ the first $N-1$ lines and recall that $\left(w_{+}^{h}\right)_{j}^{\prime}(x)=$ $\left(w_{+}^{h}\right)_{j}^{\prime}(\hat{y}, \phi(\hat{y}))$, to have

$$
\begin{array}{r}
\delta_{i, j}=y_{x_{j}}^{i}+\frac{1}{h^{2}}\left[\left(\left(w_{+}^{h}\right)_{x_{j}}-\sum_{l}\left\langle\nabla u^{0}, \bar{\tau}_{l}\right\rangle \cdot y_{x_{j}}^{l}\right)\left(w_{+}^{h}\right)_{x_{i}}\right. \\
\left.+\left(w_{+}^{h}-u^{0}\right) \sum_{l}\left\langle\nabla\left(\left(w_{+}^{h}\right)_{i}^{\prime}\right), \bar{\tau}_{l}\right\rangle y_{x_{j}}^{l}\right],
\end{array}
$$

where $\left\langle\nabla\left(\left(w_{+}^{h}\right)_{i}^{\prime}\right), \bar{\tau}_{l}\right\rangle, u^{0}$ and $\left\langle\nabla u^{0}, \bar{\tau}_{l}\right\rangle$ are computed at the point $(\hat{y}, \phi(\hat{y}))$. Hence, for $i=1, \ldots, N-1$ and $j=1, \ldots, N$,

$$
\delta_{i, j}-\frac{1}{h^{2}}\left(w_{+}^{h}\right)_{i}^{\prime}\left(w_{+}^{h}\right)_{j}^{\prime}
$$

$$
=y_{x_{j}}^{i}+\frac{1}{h^{2}}\left\{\sum_{l}\left[w_{+}^{h}\left\langle\nabla\left(\left(w_{+}^{h}\right)_{i}^{\prime}\right), \bar{\tau}_{l}\right\rangle-\left(w_{+}^{h}\right)_{x_{i}}\left\langle\nabla u^{0}, \bar{\tau}_{l}\right\rangle-u^{0}\left\langle\nabla\left(\left(w_{+}^{h}\right)_{i}^{\prime}\right), \bar{\tau}_{l}\right\rangle\right] y_{x_{j}}^{l}\right\} .
$$

System (27) has the form

$$
\begin{aligned}
& \left(\begin{array}{llll}
1+\sigma_{1,1} & \sigma_{1,2} & \cdot & \sigma_{1, N} \\
\cdot & \cdot & \cdot & \cdot \\
\sigma_{N-1,1} & 1+\sigma_{N-1,2} & \cdot & \sigma_{N-1, N}
\end{array}\right)
\end{aligned}
$$

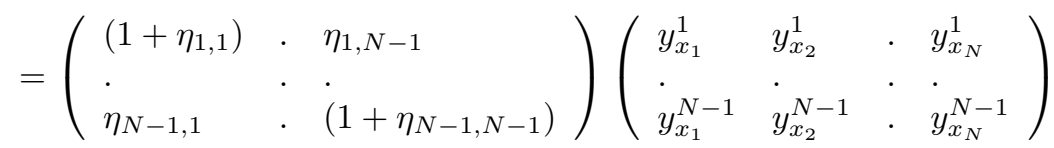

with

$$
\eta_{i, l}=\frac{1}{h^{2}}\left[w_{+}^{h}\left\langle\nabla\left(\left(w_{+}^{h}\right)_{i}^{\prime}\right), \bar{\tau}_{l}\right\rangle-\left(w_{+}^{h}\right)_{x_{i}}\left\langle\nabla u^{0}, \bar{\tau}_{l}\right\rangle-u^{0}\left\langle\nabla\left(\left(w_{+}^{h}\right)_{i}^{\prime}\right), \bar{\tau}_{l}\right\rangle\right]
$$

We claim that system (28) is solvable in the unknowns $y_{x_{j}}^{i}$; for this it is enough to show that the $\eta_{i, l}$ can be made arbitrarily small.

d) The expression for $\eta_{i, l}$ contains second derivatives of the function $w_{+}^{h}$, computed at $(\hat{y}(x), \phi(\hat{y}(x)))$, that can be obtained differentiating (25); in turn, this requires the existence of the derivatives of $a_{i}$ and of the $b_{j}^{i}$. We have the derivatives of $a_{i}$ by differentiating the identity, obtained from (22),

$$
\left\langle\nabla u^{0}(\hat{y}, \phi(\hat{y})), \bar{\tau}_{j}(\hat{y})\right\rangle \equiv \sum a_{i}(\hat{y})\left\langle\tau_{i}(\hat{y}), \bar{\tau}_{j}(\hat{y})\right\rangle
$$

we have

$$
\frac{\partial}{\partial y_{l}}\left\langle\nabla u^{0}, \bar{\tau}_{j}\right\rangle=\left(\bar{\tau}_{j}\right)^{T} H_{u^{0}} \bar{\tau}_{l}+u_{y_{N}}^{0} \phi_{y_{j} y_{l}}=\sum\left[\left(a_{i}\right)_{y_{l}}\left\langle\tau_{i}, \bar{\tau}_{j}\right\rangle+a_{i} \frac{\partial}{\partial y_{l}}\left\langle\tau_{i}, \bar{\tau}_{j}\right\rangle\right]
$$


i.e.,

$$
\left(\bar{\tau}_{j}\right)^{T} H_{u^{0}} \bar{\tau}_{l}+u_{y_{N}}^{0} \phi_{y_{j} y_{l}}-\sum_{i} a_{i} \frac{\partial}{\partial y_{l}}\left\langle\tau_{i}, \bar{\tau}_{j}\right\rangle=\sum_{i}\left(a_{i}\right)_{y_{l}}\left\langle\tau_{i}, \bar{\tau}_{j}\right\rangle
$$

Again, for all $\Phi$ sufficiently small, system (30) is solvable and $\left(a_{i}\right)_{y_{j}}$ exist.

Consider (24) and take scalar products with $\nabla u^{0}$; since the left hand side is differentiable, so is the right hand side and we obtain

$$
\left(\left\langle e_{j}, \nabla u^{0}\right\rangle\right)_{x_{l}}-\left[\left\langle\nu, e_{j}\right\rangle\left\langle\nu, \nabla u^{0}\right\rangle\right]_{x_{l}}=\sum_{r=1, \ldots, N-1}\left(b_{r}^{j}\left\langle\tau_{r}, \nabla u^{0}\right\rangle\right)_{x_{l}}
$$

Finally, consider (25); since we have shown that the right hand side is differentiable, so is the left hand side and we obtain

$$
\begin{array}{r}
\frac{\partial}{\partial y_{l}}\left\langle\nabla w_{+}^{h}, e_{j}\right\rangle(\hat{y}, \phi(\hat{y}))=\left(\left\langle e_{j}, \nu\right\rangle\right)_{y_{l}} \sqrt{h^{2}-\left(\sum_{i} a_{i}^{2}+\sum_{i \neq j} a_{i} a_{j}\left\langle\tau_{i}, \tau_{j}\right\rangle\right)} \\
+\left\langle e_{j}, \nu\right\rangle\left(\sqrt{h^{2}-\left(\sum_{i} a_{i}^{2}+\sum_{i \neq j} a_{i} a_{j}\left\langle\tau_{i}, \tau_{j}\right\rangle\right)}\right)_{y_{l}}+\sum_{i=1, \ldots, N-1}\left(b_{i}^{j}\left\langle\nabla u^{0}, \tau_{i}\right\rangle\right)_{y_{l}}
\end{array}
$$

e) Consider the following estimates as $\Phi \rightarrow 0$.

We have that, as $\Phi \rightarrow 0$, for $j=1, \ldots, N-1, \tau_{j} \rightarrow e_{j}$, while $\nu \rightarrow e_{N}$; from (22) we obtain

so that,

$$
a_{j} \rightarrow\left\langle\nabla u^{0}(\hat{0}, 0), e_{j}\right\rangle=u_{y_{j}}^{0}(\hat{0}, 0)
$$

$$
\left\langle\nabla w_{+}^{h}, \nu\right\rangle \rightarrow \sqrt{h^{2}-\sum_{i}\left(\left\langle\nabla u^{0}, e_{i}\right\rangle\right)^{2}}
$$

and

$$
\sqrt{h^{2}-\left(\sum_{i} a_{i}^{2}+\sum_{i \neq j} a_{i} a_{j}\left\langle\tau_{i}, \tau_{j}\right\rangle\right)} \rightarrow \sqrt{h^{2}-\sum_{i=1}^{N-1}\left(\left\langle\nabla u^{0}, e_{i}\right\rangle\right)^{2}}
$$

We also have

$$
\left(\left\langle e_{j}, \nu\right\rangle\right) \rightarrow \begin{cases}0 & \text { when } j \neq N \\ 1 & \text { when } j=N\end{cases}
$$

and, from (24), we obtain $b_{j}^{i} \rightarrow \delta_{i j}$; moreover,

$$
\begin{gathered}
\left(\left\langle e_{j}, \nu\right\rangle\right)_{y_{l}} \rightarrow \begin{cases}-\phi_{y_{j} y_{l}} & \text { when } j \neq N \\
0 & \text { when } j=N\end{cases} \\
\left\langle\nu, \nabla u^{0}\right\rangle \rightarrow u_{y_{N}}^{0} \text { and }\left(\left\langle\nu, \nabla u^{0}\right\rangle\right)_{y_{l}} \rightarrow-\sum_{i=1, \ldots, N-1} \phi_{y_{i} y_{l}} u_{y_{i}}^{0}+u_{y_{N} y_{l}}^{0}
\end{gathered}
$$

From (25) we infer

$$
\left(w_{+}^{h}\right)_{x_{j}}=\left\langle\nabla w_{+}^{h}, e_{j}\right\rangle \rightarrow \begin{cases}\left\langle\nabla u^{0}, e_{j}\right\rangle & \text { for } j<N \\ \sqrt{h^{2}-\sum_{i}\left\langle\nabla u^{0}, e_{i}\right\rangle^{2}} & \text { for } j=N\end{cases}
$$

From $\frac{\partial}{\partial y_{l}} \frac{\phi_{y_{i}}}{\sqrt{1+\phi_{y_{i}}^{2}}} \rightarrow \phi_{y_{i} y_{l}}$ we infer that $\frac{\partial}{\partial y_{l}}\left\langle\tau_{i}, \tau_{j}\right\rangle \rightarrow 0$; hence, solving system (30), we obtain

$$
\left(a_{j}\right)_{y_{l}} \rightarrow\left(H_{u^{0}}\right)_{j, l}+u_{y_{N}}^{0} \phi_{y_{j} y_{l}}
$$


that implies that there exists $H_{1}$ such that, for all sufficiently small $\Phi$ and all $h$, $\left|\left(a_{j}\right)_{y_{l}}\right| \leq H_{1}$. Hence, there exists $H_{2}$ such that

$$
\begin{gathered}
\frac{\partial}{\partial y_{l}} \sqrt{h^{2}-\left(\sum_{i} a_{i}^{2}+\sum_{i \neq j} a_{i} a_{j}\left\langle\tau_{i}, \tau_{j}\right\rangle\right)} \\
=\frac{\sum_{i} 2 a_{i}\left(a_{i}\right)_{y_{l}}+\sum_{i \neq j}\left[\left(a_{i} a_{j}\right)_{y_{l}}\left\langle\tau_{i}, \tau_{j}\right\rangle+a_{i} a_{j}\left(\left\langle\tau_{i}, \tau_{j}\right\rangle\right)_{y_{l}}\right]}{2 \sqrt{h^{2}-\left(\sum_{i} a_{i}^{2}+\sum_{i \neq j} a_{i} a_{j}\left\langle\tau_{i}, \tau_{j}\right\rangle\right)}} \leq H_{2}
\end{gathered}
$$

From (31) we obtain

$$
\sum_{r=1, \ldots, N-1}\left(b_{r}^{j}\left\langle\tau_{r}, \nabla u^{0}\right\rangle\right)_{y_{l}} \rightarrow \begin{cases}u_{y_{j} y_{l}}^{0}+\phi_{y_{j} y_{l}} u_{y_{N}}^{0} & j \neq N \\ \sum_{i} \phi_{y_{i} y_{l}} u_{y_{i}}^{0} & j=N\end{cases}
$$

that yields the existence of $H_{3}$ such that, for all $\Phi$ sufficiently small,

$$
\left|\sum_{r=1, \ldots, N-1}\left(b_{r}^{j}\left\langle\tau_{r}, \nabla u^{0}\right\rangle\right)_{y_{l}}\right| \leq H_{3}
$$

Then, from (32),

$$
\left|\frac{\partial}{\partial y_{l}}\left\langle\nabla w_{+}^{h}, e_{j}\right\rangle(\hat{y}, \phi(\hat{y}))\right| \leq 2 K h+H_{2}+H_{3}
$$

Since $\left|\left(w_{+}^{h}\right)_{x_{j}}\right| \leq h$, on the set $\Omega_{\frac{3 M}{h}}$ we obtain

$$
\left|\eta_{i, l}\right| \leq \frac{1}{h^{2}}\left[6 M\left(2 K h+H_{2}+H_{3}\right)+2 K h\right] .
$$

f) Consider system (27) and notice that $i<N$ : from (33) we obtain that each $\sigma_{i, j}$ can be made arbitrarily small by choosing $\frac{1}{h}$ and $\Phi$ small. From (27) we obtain that, as both $\Phi$ and $\frac{1}{h} \rightarrow 0, y_{x_{j}}^{i} \rightarrow \delta_{i j}$, with $i=1, \ldots, N-1$ and $j=1, \ldots, N$. The determinant of the minor of the matrix $\left(y_{x_{j}}^{i}\right)$ obtained by suppressing the last column, $\left(y_{x_{N}}^{i}\right)$, converges to 1 , while the determinants of all the other square matrices, that must contain the last column, tend to 0 . Hence, by the formula for the Jacobian ([6], p. 89), given $\eta$, we can find $\tilde{h} \geq h^{*}$ and $\tilde{\Phi}$ such that $h \geq \tilde{h}$ and $\Phi \leq \tilde{\Phi}$ imply that, for $x \in \Omega_{\frac{3 M}{h}}$,

$$
\frac{1-\eta}{1+\eta} \leq J(\hat{y}(x)) \leq \frac{\sqrt{1+\eta^{2}}}{(1-\eta)}
$$

\section{Proof of Theorem 1}

The Proof of Theorem 1 is partially based on the following fact: the problem of minimizing

$$
\int_{a}^{b} L\left(\left|u^{\prime}(t)\right|\right) d t
$$

on the set of $u:[a, b] \rightarrow \mathbb{R}^{N}$ absolutely continuous and satisfying $u(a)=\alpha ; u(b)=$ $\beta$, where $L$ is a convex function defined on $\mathbb{R}$, admits the solution

$$
\tilde{u}(t)=\alpha+\frac{\beta-\alpha}{b-a}(t-a) .
$$


We shall need the following Definitions. In it, and for the remainder of this section, for $\xi \in B_{\Phi}(P)$, we set $y_{\xi}=\left(\begin{array}{l}\xi \\ \phi(\xi)\end{array}\right)$.

Definition 2. For given $h, \Phi, \delta$, and for $P \in \partial \Omega$, set,

$$
\begin{gathered}
V_{h, \Phi, \delta}^{+}(P)= \\
\left\{x \in \Omega: x=y_{\xi}+\ell \frac{\nabla w_{+}^{h}\left(y_{\xi}\right)}{h} ; \xi \in B_{\Phi}(P) ; \ell \in\left(0, \ell^{*}\right) ; d\left(y_{\xi}+\ell^{*} \frac{\nabla w_{+}^{h}\left(y_{\xi}\right)}{h}\right)=\delta\right\} .
\end{gathered}
$$

For a measurable subset $Z$ of the ball $B_{\Phi}(P)$, set $V_{Z}^{+}$to be the subset of $V_{h, \Phi, \delta}^{+}(P)$ such that $\xi \in Z$.

Set

$$
\begin{gathered}
V_{h, \Phi, \delta}^{-}(P)= \\
\left\{x \in \Omega: x=y_{\xi}-\ell \frac{\nabla w_{-}^{h}\left(y_{\xi}\right)}{h} ; \xi \in B_{\Phi}(P) ; \ell \in\left(0, \ell^{*}\right) ; d\left(y_{\xi}-\ell^{*} \frac{\nabla w_{-}^{h}\left(y_{\xi}\right)}{h}\right)=\delta\right\} .
\end{gathered}
$$

For a measurable subset $Z$ of the ball $B_{\Phi}(P)$, set $V_{Z}^{-}$to be the subset of $V_{h, \Phi, \delta}^{-}(P)$ such that $\xi \in Z$.

Proof of Theorem 1. Fix $\varepsilon$. Set $\varepsilon^{1}=\frac{\varepsilon}{4 \int_{\Omega} L(|\nabla u(x)|) \mathrm{d} x}$ and let $\eta(0<\eta<1)$ be such that

$$
\frac{(1+\eta) \sqrt{1+\eta^{2}}}{(1-\eta)^{2}}=\left(1+\varepsilon^{1}\right)
$$

consider $\tilde{h}$, and $\tilde{\Phi}$ supplied by the Differentiability Lemma for this $\eta$; set $\tilde{\delta}=\frac{3 M}{\tilde{h}}$; recall the function $M^{\tilde{h}}$ in Definition 1 .

a) Set $\Omega^{+}=\left\{x: u(x)>w_{+}^{\tilde{h}}(x)\right\}, \Omega^{-}=\left\{x: u(x)<w_{-}^{\tilde{h}}(x)\right\}$ and $\Omega^{0}=$ $\left\{x: w_{-}^{\tilde{h}}(x) \leq u(x) \leq w_{+}^{\tilde{h}}(x)\right\}$. Notice that $d(x) \geq \tilde{\delta}$ implies that $w_{+}^{\tilde{h}}(x)=$ $u^{0}(y(x))+w_{+}^{\tilde{h}}(x)-u^{0}(y(x)) \geq-M+\tilde{h}|y(x)-x| \geq 2 M>M \geq u(x)$, so that $\min \left\{w_{+}^{h}(x), u(x)\right\}=u(x)$ and $\Omega^{+} \subset \Omega_{\tilde{\delta}}$. In the same way one obtains also $\Omega^{-} \subset \Omega_{\tilde{\delta}}$. Hence, the estimates on the Jacobian of the map $x \rightarrow \hat{y}$, provided by the differentiability Lemma, hold on $\Omega^{+}$and on $\Omega^{-}$.

We have, almost everywhere in $\Omega$,

$$
\left|\nabla M^{\tilde{h}}\right|= \begin{cases}\tilde{h} & \text { for } x \in \Omega^{-} \cup \Omega^{+} \\ |\nabla u| & \text { for } x \in \Omega^{0}\end{cases}
$$

so that

$$
\int_{\Omega} L\left(\left|\nabla M^{\tilde{h}}(x)\right|\right) \mathrm{d} x=\int_{\Omega^{-}} L(\tilde{h}) \mathrm{d} x+\int_{\Omega^{+}} L(\tilde{h}) \mathrm{d} x+\int_{\Omega^{0}} L(|\nabla u|) \mathrm{d} x
$$

b) We wish to show that

$$
\int_{\Omega} L\left(\left|\nabla M^{\tilde{h}}(x)\right|\right) \mathrm{d} x \leq \int_{\Omega} L(|\nabla u(x)|) \mathrm{d} x+\frac{\varepsilon}{2}
$$

it is enough to show that

$$
\int_{\Omega^{+}} L(|\tilde{h}|) \mathrm{d} x=\int_{\Omega^{+}} L\left(\left|\nabla M^{\tilde{h}}(x)\right|\right) \mathrm{d} x \leq \int_{\Omega^{+}} L(|\nabla u(x)|) \mathrm{d} x+\frac{\varepsilon}{4}
$$

and

$$
\int_{\Omega^{-}} L(|\tilde{h}|) \mathrm{d} x=\int_{\Omega^{-}} L\left(\left|\nabla M^{\tilde{h}}(x)\right|\right) \mathrm{d} x \leq \int_{\Omega^{-}} L(|\nabla u(x)|) \mathrm{d} x+\frac{\varepsilon}{4} .
$$


c) We hall prove (35), being (36) proved in the same way. Consider $\Delta=\{x \in$ $\left.\Omega: d(x)=\frac{\tilde{\delta}}{2}\right\}: \Delta$ is a compact subset of $\Omega$. By ii) of Lemma 1 , the collection of open sets, defined in Definition 2, $\left\{V_{\tilde{h}, \tilde{\Phi}, \tilde{\delta}}^{+}(P): P \in \partial \Omega\right\}$ is a covering of $\Delta$. Let $\left\{V_{\tilde{h}, \tilde{\Phi}, \tilde{\delta}}^{+}\left(P_{j}\right): 1 \leq j \leq J\right\}$ be a finite subcover. We are going to define measurable subsets $Z_{j}$ of $B_{\tilde{\Phi}}\left(P_{j}\right)$ : set $Z=Z_{1}=B_{\tilde{\Phi}}\left(P_{1}\right)$; consider $P_{2}$ and set

$$
Z_{2}=\left\{\xi \in B_{\tilde{\Phi}}\left(P_{2}\right):\left(y_{\xi}+\frac{\tilde{\delta}}{2} \frac{\nabla w_{+}^{\tilde{h}}\left(y_{\xi}\right)}{\tilde{h}}\right) \cap V_{Z_{1}}^{+}=\emptyset\right\} .
$$

Having defined $Z_{j}$ up to $\tilde{j}$, set

$$
Z_{\tilde{j}+1}=\left\{\xi \in B_{\tilde{\Phi}}\left(P_{\tilde{j}+1}\right):\left(y_{\xi}+\frac{\tilde{\delta}}{2} \frac{\nabla w_{+}^{\tilde{h}}\left(y_{\xi}\right)}{\tilde{h}}\right) \cap V_{Z_{j}}^{+}=\emptyset \text { for } 1 \leq j \leq \tilde{j}\right\} ;
$$

Hence, every point in $\Delta$ belongs to one and only one $V_{Z_{j}}^{+}$and, by the uniqueness in Lemma 1, so is for $\Omega_{\tilde{\delta}}$.

d) We claim that for every $j$,

$$
\int_{\Omega^{+} \cap V_{Z_{j}^{+}}^{+}} L\left(\left|\nabla M^{\tilde{h}}(x)\right|\right) \mathrm{d} x \leq(1+\varepsilon) \int_{\Omega^{+} \cap V_{Z_{j}^{+}}^{+}} L(|\nabla u(x)|) \mathrm{d} x .
$$

Apply the coarea theorem [6] to the set $\Omega^{+} \cap V_{Z_{j}}^{+}$and to the function $\hat{y}(x)$ to obtain

$$
\int_{\Omega^{+} \cap V_{Z_{j}}^{+}} L(|\nabla u(x)|) \mathrm{d} x=\int_{Z_{j}+}\left[\int_{\{\hat{y}(x)=\xi\} \cap\left(\Omega^{+} \cap V_{Z_{j}}^{+}\right)} \frac{L(|\nabla u(x)|)}{J(\hat{y}(x))} \mathrm{d} H^{1}\right] \mathrm{d} \xi ;
$$

consider the line segment

$$
L_{\xi}=\left\{y_{\xi}+\ell \frac{\nabla w_{+}^{\tilde{h}}\left(y_{\xi}\right)}{\tilde{h}}: \ell \in\left(0, \ell^{*}\right) ; d\left(y_{\xi}+\ell^{*} \frac{\nabla w_{+}^{\tilde{h}}\left(y_{\xi}\right)}{\tilde{h}}\right)=\tilde{\delta}\right\}:
$$

we have that $\{\hat{y}(x)=\xi\} \cap\left(\Omega^{+} \cap V_{Z_{j}}\right)=L_{\xi} \cap \Omega^{+}$. For almost every $\xi \in Z_{j}$ the maps

$$
\begin{gathered}
\tilde{u}_{\xi}(\ell)=u\left(y_{\xi}+\ell \frac{\nabla w_{+}^{\tilde{h}}\left(y_{\xi}\right)}{\tilde{h}}\right), \\
\tilde{w}_{+}^{\tilde{h}}(\ell)=w_{+}^{\tilde{h}}\left(y_{\xi}+\ell \frac{\nabla w_{+}^{\tilde{h}}\left(y_{\xi}\right)}{\tilde{h}}\right)
\end{gathered}
$$

are absolutely continuous, so that the set $S_{\xi}=\left\{\ell: \tilde{u}_{\xi}(\ell)>\tilde{w}_{+}^{\tilde{h}}(\ell)\right\}$ is a (possibly empty) open set. Then, there are at most countably many open intervals $\left(a_{j}, b_{j}\right)$ such that $S_{\xi}=\cup\left(a_{j}, b_{j}\right)$ and $\tilde{u}_{\xi}\left(a_{j}\right)-\tilde{w}_{+}^{\tilde{h}}\left(a_{j}\right)=\tilde{u}_{\xi}\left(b_{j}\right)-\tilde{w}_{+}^{\tilde{h}}\left(b_{j}\right)=0$ while, for $\ell \in\left(a_{j}, b_{j}\right), \tilde{u}_{\xi}(\ell)>\tilde{w}_{+}^{\tilde{h}}(\ell)$. Fix one such $\left(a_{j}, b_{j}\right)$. The problem of minimizing

$$
\int_{a_{j}}^{b_{j}} L\left(\left|v^{\prime}(\ell)\right|\right) \mathrm{d} \ell ; \quad v\left(a_{j}\right)=\tilde{u}_{\xi}\left(a_{j}\right) ; v\left(b_{j}\right)=\tilde{u}_{\xi}\left(b_{j}\right)
$$

admits the solution $\tilde{w}_{+}^{\tilde{h}}$, so that, in particular,

$$
\int_{a_{j}}^{b_{j}} L(\tilde{h}) \mathrm{d} \ell \leq \int_{a_{j}}^{b_{j}} L\left(\left|\tilde{u}_{\xi}^{\prime}(\ell)\right|\right) \mathrm{d} \ell=\int_{a_{j}}^{b_{j}} L\left(\left|\left\langle\nabla u\left(y_{\xi}+\ell \frac{\nabla w_{+}^{\tilde{h}}\left(y_{\xi}\right)}{\tilde{h}}\right), \frac{\nabla w_{+}^{\tilde{h}}\left(y_{\xi}\right)}{\tilde{h}}\right\rangle\right|\right) \mathrm{d} \ell .
$$


Recall that $\left|\frac{\nabla w^{\tilde{h}}\left(y_{\xi}\right)}{\tilde{h}}\right|=1$; since $L$ is non-decreasing, we obtain that

$$
L\left(\left|\left\langle\nabla u\left(y_{\xi}+\ell \frac{\nabla w_{+}^{\tilde{h}}\left(y_{\xi}\right)}{\tilde{h}}\right), \frac{\nabla w_{+}^{\tilde{h}}\left(y_{\xi}\right)}{\tilde{h}}\right\rangle\right|\right) \leq L\left(\left|\nabla u\left(y_{\xi}+\ell \frac{\nabla w_{+}^{\tilde{h}}\left(y_{\xi}\right)}{\tilde{h}}\right)\right|\right),
$$

hence that

$$
\int_{a_{j}}^{b_{j}} L(\tilde{h}) \mathrm{d} \ell \leq \int_{a_{j}}^{b_{j}} L\left(\mid \nabla u\left(y_{\xi}+\ell \frac{\nabla w_{+}^{\tilde{h}}\left(y_{\xi}\right)}{\tilde{h}} \mid\right) \mathrm{d} \ell\right.
$$

Since the restriction to $L_{\xi} \cap \Omega^{+}$of the gradient of $M^{\tilde{h}}$ is $\nabla w_{+}^{\tilde{h}}\left(y_{\xi}\right)$, hence $\left|\nabla M^{\tilde{h}}\right|=\tilde{h}$, when $\ell$ belongs to the intervals $\left(a_{j}, b_{j}\right)$, inequality $(39)$ implies

$$
\int_{\{\hat{y}(x)=\xi\} \cap\left(V_{Z_{j}} \cap \Omega^{+}\right)} L\left(\left|\nabla M^{\tilde{h}}\right|\right) \mathrm{d} H^{1} \leq \int_{\{\hat{y}(x)=\xi\} \cap\left(V_{Z_{j}} \cap \Omega^{+}\right)} L(|\nabla u|) \mathrm{d} H^{1} .
$$

By (37), (18) and (40),

$$
\begin{gathered}
\int_{V_{Z_{j}} \cap \Omega^{+}} L(|\nabla u(x)|) \mathrm{d} x=\int_{Z_{j}}\left[\int_{\{\hat{y}(x)=\xi\} \cap\left(V_{Z_{j}} \cap \Omega^{+}\right)} \frac{L(|\nabla u(x)|)}{J(\hat{y}(x))} \mathrm{d} H^{1}\right] \mathrm{d} \xi \\
\geq \frac{(1-\eta)}{\sqrt{1+\eta^{2}}} \int_{Z_{j}}\left[\int_{\{\hat{y}(x)=\xi\} \cap\left(V_{Z_{j}} \cap \Omega^{+}\right)} L(|\nabla u(x)|) \mathrm{d} H^{1}\right] \mathrm{d} \xi \\
\geq \frac{(1-\eta)}{\sqrt{1+\eta^{2}}} \int_{Z_{j}}\left[\int_{\{\hat{y}(x)=\xi\} \cap\left(V_{Z_{j}} \cap \Omega^{+}\right)} L\left(\left|\nabla M^{\tilde{h}}(x)\right|\right) \mathrm{d} H^{1}\right] \mathrm{d} \xi \\
\geq \frac{(1-\eta)}{\sqrt{1+\eta^{2}}} \int_{Z_{j}}\left[\int_{\{\hat{y}(x)=\xi\} \cap\left(V_{Z_{j}} \cap \Omega^{+}\right)} \frac{1-\eta}{1+\eta} \frac{L\left(\left|\nabla M^{\tilde{h}}(x)\right|\right)}{J(\hat{y}(x))} \mathrm{d} H^{1}\right] \mathrm{d} \xi \\
=\frac{(1-\eta)^{2}}{(1+\eta) \sqrt{1+\eta^{2}}} \int_{V_{Z_{j}} \cap \Omega^{+}} L\left(\left|\nabla M^{\tilde{h}}(x)\right|\right) \mathrm{d} x .
\end{gathered}
$$

We have obtained

$$
\int_{V_{Z_{j}} \cap \Omega^{+}} L\left(\left|\nabla M^{\tilde{h}}(x)\right|\right) \mathrm{d} x \leq\left(1+\varepsilon^{1}\right) \int_{V_{Z_{j}} \cap \Omega^{+}} L(|\nabla u(x)|) \mathrm{d} x .
$$

Summing over $j$, we have

$$
\int_{\Omega^{+}} L\left(\left|\nabla M^{\tilde{h}}(x)\right|\right) \mathrm{d} x \leq\left(1+\varepsilon^{1}\right) \int_{\Omega^{+}} L(|\nabla u(x)|) \mathrm{d} x \leq \int_{\Omega^{+}} L(|\nabla u(x)|) \mathrm{d} x+\frac{\varepsilon}{2},
$$

thus (34) is proved.

e) Write

$$
M^{\tilde{h}}(x)=u^{0}(x)+\left(M^{\tilde{h}}(x)-M^{\tilde{h}}(y(x))-\left(u^{0}(x)-u^{0}(y(x))\right) ;\right.
$$

we have $\mid\left(M^{\tilde{h}}(x)-M^{\tilde{h}}(y(x))-\left(u^{0}(x)-u^{0}(y(x))\right)|\leq(\tilde{h}+K)| y(x)-x \mid \leq 2(\tilde{h}+K) d(x)\right.$ by i) of Lemma 1 . Hence, $M^{\tilde{h}}$ is the sum of a Lipschitzean function and of a function $g$ such that $|g(x)| \leq \operatorname{Dd}(x)$.

Apply Lemma 3 to infer the existence of $D^{*}$ such that

$$
\tilde{M}^{\tilde{h}}(x)=\frac{1}{|B(x, d(x))|} \int_{B(x, d(x))} M^{\tilde{h}}(z) \mathrm{d} z
$$


is Lipschitzean of constant $D^{*}$. Consider $L\left(D^{*}\right)$ : there exists $\delta^{*} \leq \tilde{\delta}$ such that

$$
\int_{\Omega_{\delta^{*}}} L\left(D^{*}\right) \mathrm{d} x<\frac{\varepsilon}{2} .
$$

f) Having fixed $\delta^{*}$, define the continuous function

$$
u_{\varepsilon}(x)=\left\{\begin{array}{cc}
\frac{1}{|B(x, d(x))|} \int_{B(x, d(x))} M^{\tilde{h}}(z) \mathrm{d} z, & \text { when } d(x) \leq \delta^{*} \\
\frac{1}{\left|B\left(x, \delta^{*}\right)\right|} \int_{B\left(x, \delta^{*}\right)} M^{\tilde{h}}(z) \mathrm{d} z, & \text { when } d(x)>\delta^{*} .
\end{array}\right.
$$

From e) and Lemma 2, we have that $u_{\varepsilon}$ is Lipschitzean and, moreover, that $\left.u_{\varepsilon}\right|_{\partial \Omega}=$ $\left.u^{0}\right|_{\partial \Omega}$. We claim that

$$
\int_{\Omega} L\left(\left|\nabla u_{\varepsilon}(x)\right|\right) \mathrm{d} x \leq \int_{\Omega} L\left(\left|\nabla M^{\tilde{h}}\right|\right) \mathrm{d} x+\frac{\varepsilon}{2} .
$$

Write $\Omega=\Omega_{\delta^{*}} \cup\left[\Omega \backslash \Omega_{\delta^{*}}\right]$. Consider the restriction of $u_{\varepsilon}$ to $\Omega \backslash \Omega_{\delta^{*}}$. By ii) of Lemma 2 (applied to $\delta=\delta^{*}$ ) we have that, for a.e. $x \in \Omega \backslash \Omega_{\delta^{*}}$,

$$
\nabla u_{\varepsilon}(x)=\frac{1}{\omega_{N}\left(\delta^{*}\right)^{N}} \int_{B\left(0, \delta^{*}\right)} \nabla M^{\tilde{h}}(x-z) \mathrm{d} z
$$

so that, by the convexity of $L(|\cdot|)$,

and

$$
L\left(\left|\nabla u_{\varepsilon}(x)\right|\right) \leq \frac{1}{\omega_{N}\left(\delta^{*}\right)^{N}} \int_{B\left(0, \delta^{*}\right)} L\left(\left|\nabla M^{\tilde{h}}(x-z)\right|\right) \mathrm{d} z
$$

$$
\begin{aligned}
\int_{\Omega \backslash \Omega_{\delta^{*}}} L\left(\left|\nabla u_{\varepsilon}(x)\right|\right) \mathrm{d} x \leq & \frac{1}{\omega_{N}\left(\delta^{*}\right)^{N}} \int_{B\left(0, \delta^{*}\right)}\left[\int_{\Omega \backslash \Omega_{\delta^{*}}} L\left(\left|\nabla M^{\tilde{h}}(x-z)\right|\right) \mathrm{d} x\right] \mathrm{d} z \\
& \leq \int_{\Omega} L\left(\left|\nabla M^{\tilde{h}}(x)\right|\right) \mathrm{d} x .
\end{aligned}
$$

By Lemma $3, u_{\varepsilon}$ is Lipschitzean of constant $D^{*}$; from our choice of $\delta^{*}$, we have

we have proved

$$
\int_{\Omega_{\delta^{*}}} L\left(\left|\nabla u_{\varepsilon}(x)\right|\right) \mathrm{d} x \leq \int_{\Omega_{\delta^{*}}} L\left(D^{*}\right) \mathrm{d} x \leq \frac{\varepsilon}{2}
$$

$$
\begin{aligned}
\int_{\Omega} L\left(\left|\nabla u_{\varepsilon}(x)\right|\right) d x= & \int_{\Omega_{\delta^{*}}} L\left(\left|\nabla u_{\varepsilon}(x)\right|\right) \mathrm{d} x+\int_{\Omega \backslash \Omega_{\delta^{*}}} L\left(\left|\nabla u_{\varepsilon}(x)\right|\right) \mathrm{d} x \\
& \leq \int_{\Omega} L\left(\left|\nabla M^{\tilde{h}}\right|\right) \mathrm{d} x+\frac{\varepsilon}{2}
\end{aligned}
$$

thus, by (34), proving the Theorem.

\section{A two-dimensional Manià-type example}

Set $\Omega$ be the square $Q=[-1 / 2,1 / 2] \times[0,1] \subset \mathbb{R}^{2}$ and let $u^{0}(x, y)=y$ be the boundary data. We wish to show the occurrence of the Lavrentiev phenomenon, i.e., that

$$
\inf _{v \in \mathcal{W}_{1}} \int_{Q} f(x, y, v, \nabla v) \mathrm{d} x \mathrm{~d} y<\inf _{v \in \mathcal{W}_{\infty}} \int_{Q} f(x, y, v, \nabla v) \mathrm{d} x \mathrm{~d} y
$$

where

$$
f(x, y, u, \nabla u):=\left\{[(1-2|x|) \sqrt[3]{y}+2|x| y]^{3}-u^{3}(x, y)\right\}^{2}\left\{\frac{\partial u}{\partial y}(x, y)\right\}^{6},
$$


and $\mathcal{W}_{p}=\left\{u \in W^{1, p}(Q):\left.u\right|_{\partial Q}=u^{0}\right\}$, for $p \in[1, \infty]$. As one can easily see, the minimum over $\mathcal{W}_{1}$ is non negative and it is attained at $u(x, y)=(1-2|x|) \sqrt[3]{y}+2|x| y$.

In order to prove (42), we adapt the original proof by B. Manià, [8], to the two-dimensional case.

Let $u$ be in $\mathcal{W}_{\infty}$. By regularity, for any fixed $x>0$, one can choose $\alpha=\alpha(x)$ and $\beta=\beta(x)$ such that $\alpha(x)<\beta(x)$ and

$$
\begin{aligned}
& u(x, \alpha(x))=\frac{1}{4}[(1-2 x) \sqrt[3]{\alpha(x)}+2 x \alpha(x)] \\
& u(x, \beta(x))=\frac{1}{2}[(1-2 x) \sqrt[3]{\beta(x)}+2 x \beta(x)]
\end{aligned}
$$

Moreover, if one considers $x \in[1 / 8,1 / 4]$, then

$$
\begin{aligned}
u(x, \beta(x))-u(x, \alpha(x)) & =\frac{1}{2}[(1-2 x) \sqrt[3]{\beta}+2 x \beta]-\frac{1}{4}[(1-2 x) \sqrt[3]{\alpha}+2 x \alpha] \\
& \geq \frac{1}{4} \sqrt[3]{\beta(x)}+\frac{1}{8} \beta(x)-\frac{3}{16} \sqrt[3]{\alpha(x)}-\frac{1}{8} \alpha(x) \\
& \geq \frac{1}{16} \sqrt[3]{\beta(x)}
\end{aligned}
$$

Using Jensen's inequality and the fact that $\beta(\cdot)<1$,

$$
\begin{array}{rl}
\int_{1 / 8}^{1 / 4} & \mathrm{~d} x \int_{\alpha(x)}^{\beta(x)}\left\{[(1-2 x) \sqrt[3]{y}+2 x y]^{3}-u^{3}(x, y)\right\}^{2}\left\{\frac{\partial u}{\partial y}(x, y)\right\}^{6} \mathrm{~d} y \\
& \geq \int_{1 / 8}^{1 / 4} \mathrm{~d} x \int_{\alpha(x)}^{\beta(x)}\left\{[(1-2 x) \sqrt[3]{y}+2 x y]^{3}-\frac{1}{8}[(1-2 x) \sqrt[3]{y}+2 x y]^{3}\right\}^{2}\left\{\frac{\partial u}{\partial y}\right\}^{6} \mathrm{~d} y \\
& \geq \frac{7^{2}}{8^{2}} \int_{1 / 8}^{1 / 4} \mathrm{~d} x \int_{\alpha(x)}^{\beta(x)} y^{2}\left\{\frac{\partial u}{\partial y}(x, y)\right\}^{6} \mathrm{~d} y \\
& =\frac{7^{2} 3^{5}}{8^{2} 5^{5}} \int_{1 / 8}^{1 / 4} \mathrm{~d} x \int_{\alpha^{3 / 5}(x)}^{\beta^{3 / 5}(x)}\left\{\frac{\partial u}{\partial y}(x, y(\xi))\right\}^{6} \mathrm{~d} \xi \\
& =\frac{7^{2} 3^{5}}{8^{2} 5^{5}} \int_{1 / 8}^{1 / 4} \frac{\beta^{3 / 5}(x)-\alpha^{3 / 5}(x)}{\beta^{3 / 5}(x)-\alpha^{3 / 5}(x)} \int_{\alpha^{3 / 5}(x)}^{\beta^{3 / 5}(x)}\left\{\frac{\partial u}{\partial y}(x, y(\xi))\right\}^{6} \mathrm{~d} \xi \mathrm{d} x \\
& \geq \frac{7^{2} 3^{5}}{8^{2} 5^{5}} \int_{1 / 8}^{1 / 4}\left[\beta^{3 / 5}(x)-\alpha^{3 / 5}(x)\right]\left(\frac{1}{\beta^{3 / 5}(x)-\alpha^{3 / 5}(x)} \int_{\alpha^{3 / 5}(x)}^{\beta^{3 / 5}(x)}\left\{\frac{\partial u}{\partial y}\right\} \mathrm{d} \xi\right)^{6} \mathrm{~d} x \\
\geq & \frac{7^{2} 3^{5}}{8^{2} 5^{5}} \int_{1 / 8}^{1 / 4} \frac{1}{\left[\beta^{3 / 5}(x)-\alpha^{3 / 5}(x)\right]^{5}}[u(x, \beta(x))-u(x, \alpha(x))]^{6} \mathrm{~d} x \\
\geq & \frac{7^{2} 3^{5}}{8^{2} 5^{5}} \int_{1 / 8}^{1 / 4} \frac{1}{\beta^{3}(x)}[u(x, \beta(x))-u(x, \alpha(x))]^{6} \mathrm{~d} x \\
\geq & \frac{7^{2} 3^{5}}{8^{2} 5^{5}} \frac{1}{16} \int_{1 / 8}^{1 / 4} \frac{\sqrt[3]{\beta(x)}}{\beta^{3}(x)} \mathrm{d} x \\
\geq & \frac{7^{2} 3^{5}}{8^{2} 5^{5} 2^{4}} \frac{1}{8}
\end{array}
$$




\section{REFERENCES}

[1] Alberti, G.; Serra Cassano, F. Non-occurrence of gap for one-dimensional autonomous functionals. Calculus of variations, homogenization and continuum mechanics (Marseille, 1993), 117, Ser. Adv. Math. Appl. Sci., 18, World Sci. Publ., River Edge, NJ, 1994

[2] Buttazzo, G; Belloni, M. A survey on old and recent results about the gap phenomenon in the calculus of variations. Recent developments in well-posed variational problems, 127, Math. Appl., 331, Kluwer Acad. Publ., Dordrecht, 1995.

[3] Esposito,L; Leonetti,F; Mingione,G. Regularity for minimizers of functionals with $p-q$ growth. Nonlinear Diff. Eq. Appl. 6 (1999) 133-148

[4] Mariconda, C. The lack of strict convexity and the validity of comparison principles for a simple class of minimizers Nonlinear Analysis TMA, 73 (2010) 828-834

[5] Esposito,L; Leonetti,F; Mingione,G. Sharp regularity for functionals with $(p, q)$ growth Jour. Diff. Eq. 204 (2004) 5-55

[6] Evans, L. C.; Gariepy, R. F. Measure theory and fine properties of functions. Studies in Advanced Mathematics. CRC Press, Boca Raton, FL, 1992.

[7] Lavrentiev, M. Sur quelques problèmes du calcul des variations. Ann. Mat. Pura Appl. 4, 1927, 9-28

[8] Manı̇̀, B. Sopra un esempio di Lavrentieff, Boll. Un. Matem. Ital. 13 (1934), 147-153.

Dipartimento di Matematica e Applicazioni, Università degli Studi di Milano-Bicocca, Via R. Cozzi 53, 20125 Milano

E-mail address: g.bonfanti3@campus.unimib.it

Dipartimento di Matematica e Applicazioni, Università degli Studi di Milano-Bicocca, Via R. CozZi 53, 20125 Milano

E-mail address: arrigo.cellina@unimib.it 